\title{
TENSILE PROPERTIES OF RAIL AND SOME OTHER STEELS AT ELEVATED TEMPERATURES
}

\author{
By John R. Freeman, jr., and G. Willard Quick
}

\section{ABSTRACT}

A study has been made of the tensile properties at elevated temperatures of several different heats of rail steel and some other steels. Special study was made of the ductility of the steels in the temperature range $400^{\circ}$ to $700^{\circ} \mathrm{C}$. In this temperature range it was found that certain rail and other steels showed a marked decrease in elongation and reduction of area values. This phenomenon has been termed "secondary brittleness" and the range the "secondary brittle range." Data are also given on the temperature gradients existing in a rail during cooling in air and when quenched in water. A theory is presented showing that internal failures, such as "shatter cracks" and "hair cracks," may be due to thermal stresses developed in a steel structure while cooling through the secondary brittle range.

\section{CONTENTS}

Page

I. Introduction

II. Test methods

III. Results of preliminary series of tests

1. Tensile properties of three heats of rail steel at elevated temperatures

IV. Discussion of preliminary results

V. Tensile properties of several rail steels at elevated temperatures.--

1. Transverse fissured rails...

2. Heat-treated rails

3. Medium manganese rails

4. Manganese molybdenum rail

5. Rail steel poured at high temperatures

6. Steel from a rail from a reheated bloom

VI. Tensile properties at elevated temperatures of some steels other

than rail steels.....

1. Chrome-molybdenum tire steel

2. One per cent carbon steel

3. Cast steel (0.30 per cent carbon)

VII. Effect of rate of application of stress on secondary brittleness

VIII. Effect of annealing on secondary brittleness.

IX. Nature of fracture at elevated temperatures

X. Temperature distribution in a section of 130 -pound rail under different cooling conditions

1. Test methods used.

2. Results of tests

(a) Costs 575

(b) Cooling in moving air 575

(c) Quenching in cold water. 578

(d) Interrupted quenching 582

(e) Quenching in boiling water 584

XI. Discussion of results with special reference to shatter cracks in rail and other steels.

551

552

552
556

559

559

560

562

564

565

567

567

569

570

571

572

573

\section{INTRODUCTION}

The tensile properties of steels at elevated temperatures have been studied by numerous investigators ${ }^{1}$ primarily for the purpose of determining their suitability for structural uses. Tests with this

\footnotetext{
1 Symposium on Effect of Temperature Upon the Properties of Metals, A. S. T. M., 24, Pt. II; 1924, p. 9.
} 
objective have been confined largely to the temperature range of approximately $20^{\circ}$ to $500^{\circ} \mathrm{C}$. because of the rapidly decreasing tensile strength at the latter temperature. Tests have also been extended to higher temperatures ${ }^{2}$ for the purpose of determining the most suitable forging range. This temperature range is also of theoretical interest.

TABLE 1.-Material studied

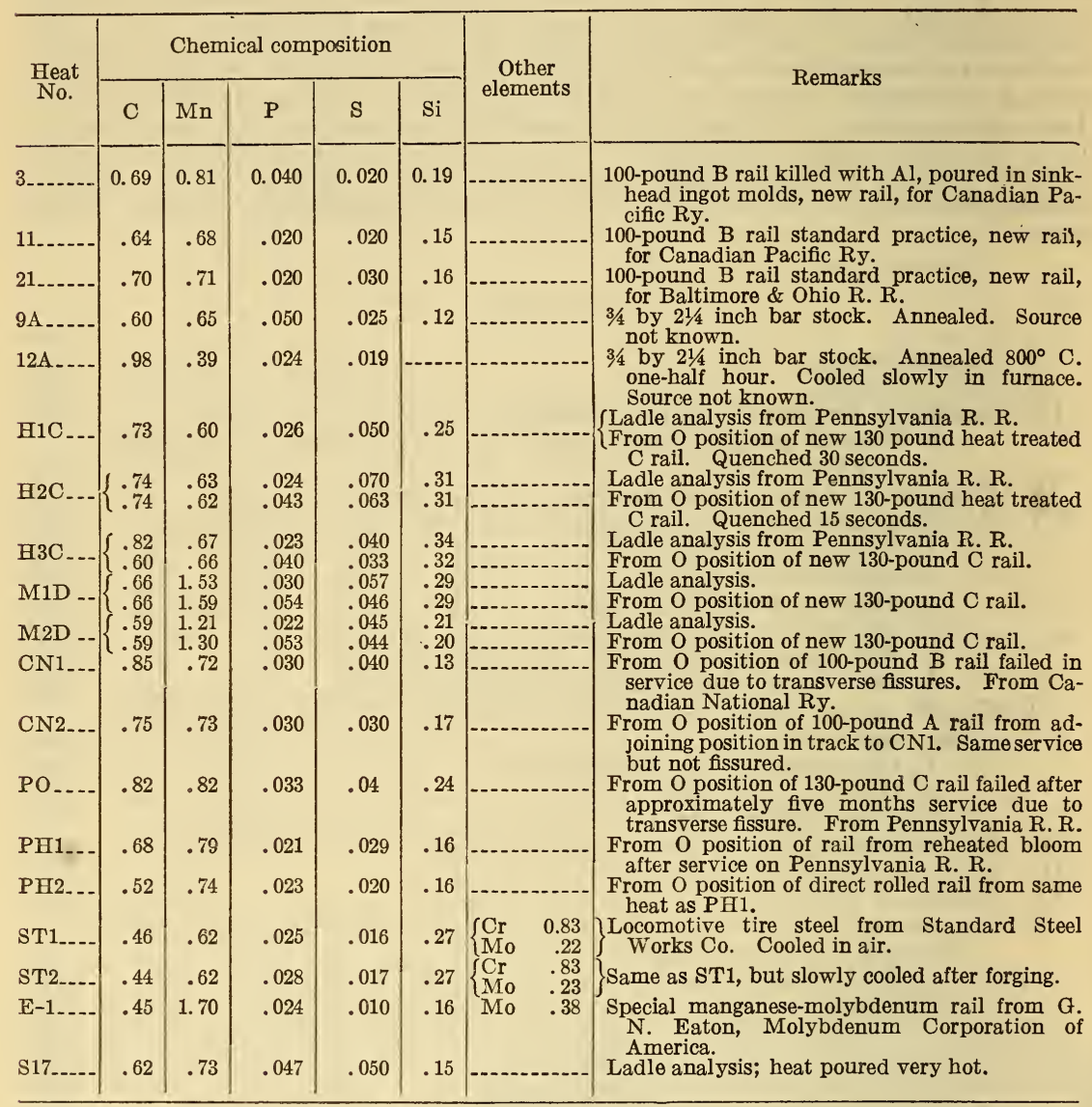

The phenomenon of increased tensile strength and decreased ductility in the so-called "blue-heat" range (approximately $200^{\circ}$ to $300^{\circ} \mathrm{C}$.) is well known. It was suggested to the bureau early in 1926 that a study of the properties of rail steels in this temperature range might prove of interest in relation to the studies of transverse fissure failures in rails. There were available from another investigation ${ }^{3}$

2 Rosenhain, W., and Humfrey, J. C. W., The Tenacity, Deformation and Fracture of Soft Steel at High Temperatures, J. Iron \& Steel Inst., 8\%, p. 219; 1913. DuPuy E., Researches expérimentales sur les Propriétés Mecaniques des acier aux Temperatures élévies., Rev. de Met., 18, p. 331; 1921; also J. Iron \& Steel Inst, 104, No. 2, p. 91; 1921. Sauveur, A., What is Steel? Howe Memorial Lecture, A. I. M. M. E., 70, p 3; 1924. Inokuty, T., Tensile Tests of Steels at High Temperatures, Sci. Repts. Tohoku Imperial Univ ; July, 1928.

${ }_{3}^{3}$ Freeman, John R. jr., Dowdell, R. L., and Berry, W. J., Endurance and Other Properties of Rail Steel, B. S. Tech. Paper No. 363; 1928 (see p. 332). 
several new rails from several different heats made to approximately the same specification requirements, the complete manufacturing histories of which were known.

It was decided to determine, at least in the preliminary series of tests, only the ultimate tensile strength, elongation and reduction of area because of the difficulty, time, and cost involved in determining the proportional limit and yield point at elevated temperatures.

A preliminary series of tests was first made on specimens from B rails from heats Nos. 3,11 , and $21{ }^{4}$ the compositions, brief history, and sources of which are given in Table 1. This preliminary series of tests was completed early in 1927, but in view of the unusual results obtained and their possible great importance in explaining the cause of certain types of failures, not only in rail but in other steels, it was considered desirable to withhold publication until a more detailed study could be made of the phenomenon discovered which has been termed secondary brittleness.

The data presented in this report are principally the results of a survey made to determine the extent to which the phenomenon exists,

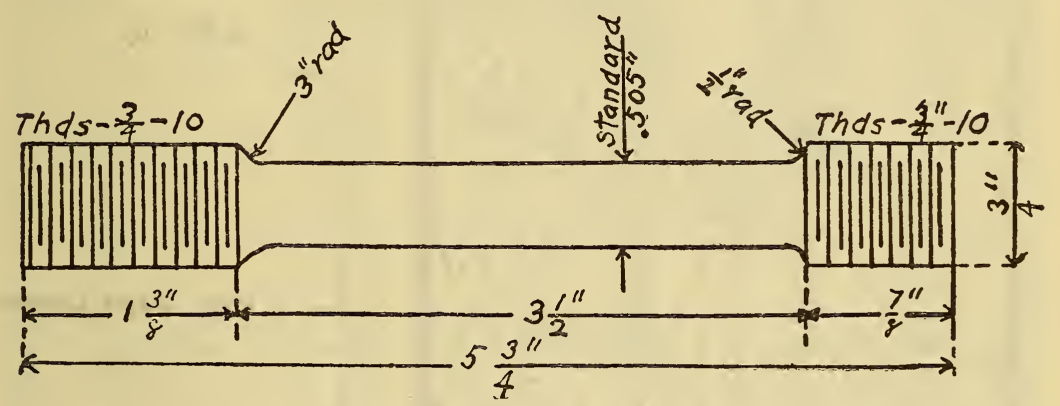

FIGURE 1.-Design of tensile test specimen used for elevated temperature tests

together with a discussion of the influence its presence in a steel may have on the final quality of the steel and are presented with the expressed purpose of obtaining the very helpful discussion and cooperation that comes from the publication of the results of studies of relatively little-known phenomena.

\section{TEST METHODS}

The design of test specimens used is given in Figure 1. A suitable furnace was designed and built for the purpose and is shown schematically in Figure 2. Two chromel-alumel thermocouples were mounted on each specimen for each test to serve as a check on the temperature gradient in the specimen. The wires of the couples were peened into small holes drilled in the specimen at the fillets. The temperature at the middle section was estimated from a calibration in which a thermocouple was also placed at the middle section, and the temperature gradient between this section and the fillets determined for the various temperatures at which tests were made. The temperatures of tests reported are believed accurate within $\pm 10^{\circ} \mathrm{C}$.

4 Throughout this report the same heat numbers are used to designate the same material, data on $\mathrm{which}$ have been given in these two previous publications. Endurance and Other Properties of Rail Steel, Freeman, John R., jr., Dowdell, R. L., and Berry, Wm., jr., B. S. Tech. Paper No. 363. Effect of Service on Endurance Properties of Rail Steel. Freeman, John R., jr., and Solakian H. N., B. S. Jour. Research; August, 1929. 
All tests were made in a 100,000-pound capacity testing machine of the screw type. A constant rate of application of stress of about 0.28 inch per minute was used in all cases except in a few special tests in which a study was made of the effect of change of rate of application of stress on the property of the steel being investigated.

\section{RESULTS OF PRELIMINARY TESTS}

\section{TENSILE PROPERTIES OF THREE HEATS OF RAIL STEEL AT ELEVATED TEMPERATURES}

The results of tensile tests of specimens taken from the head and base of a new B rail from heat No. 3 are given in Figure 3. The

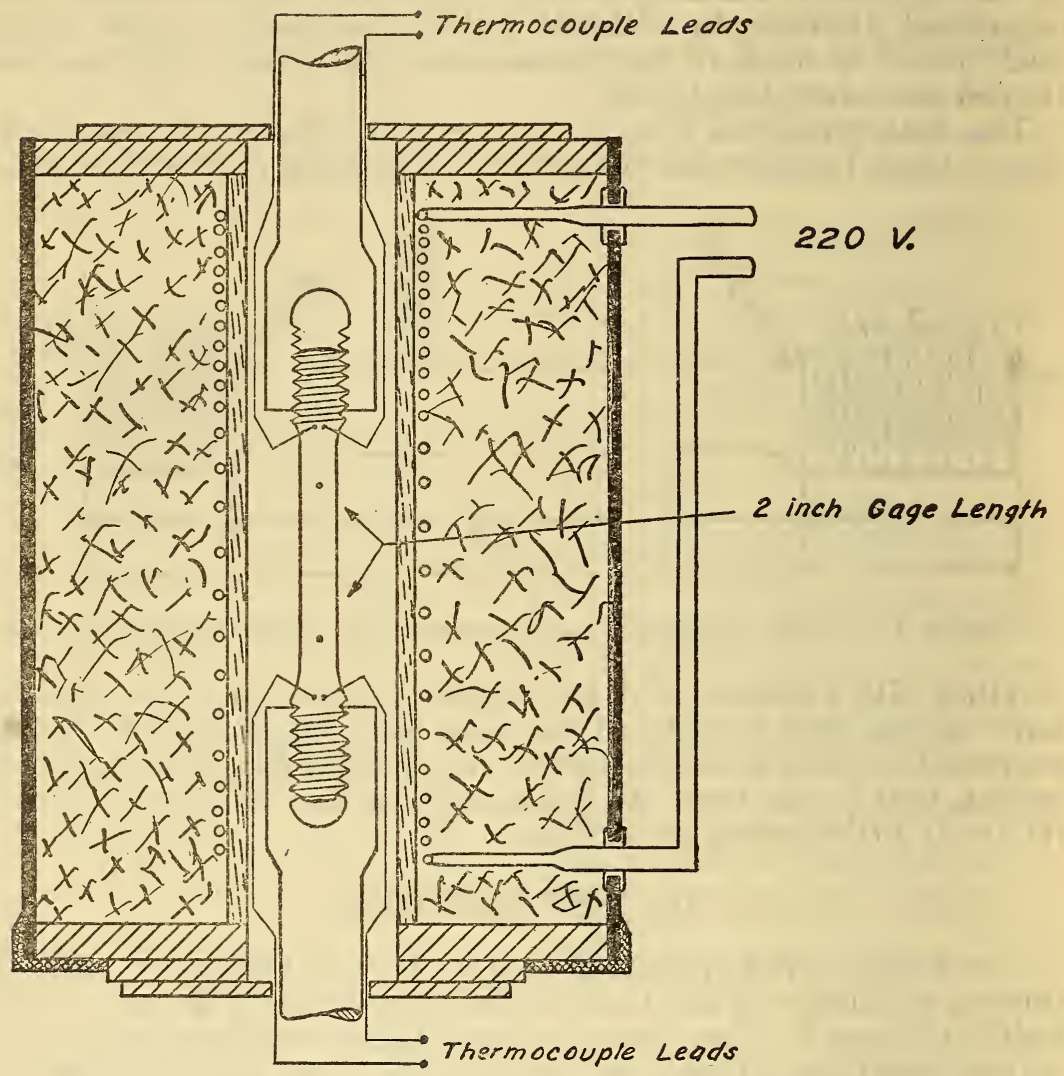

Figure 2.-Design of furnace used for elevated temperature tests

specimens from the head of rail were taken from the " $O$ " position and those from the base were taken at the junction of the web with the base of the rail which has been designated the " $F$ " position. In both cases all the specimens from each position were taken contiguously (end to end) along the length of the rail.

It is evident from the data that the tensile properties of this rail steel in the blue-heat range are characteristic of steel in this temperature range. With increase in temperature above normal $\left(20^{\circ} \mathrm{C}\right.$.) 
there is first a slight drop in tensile strength followed by an increase to a maximum value at approximately $300^{\circ} \mathrm{C}$. With further increase in temperature the tensile strength decreases nearly linearly with increasing temperature. The ductility values also indicate no unusual properties through the blue-heat range. There is apparently the usual slight decrease followed by a definite increase with increase in temperature up to about $400^{\circ} \mathrm{C}$. However, at this temperature a

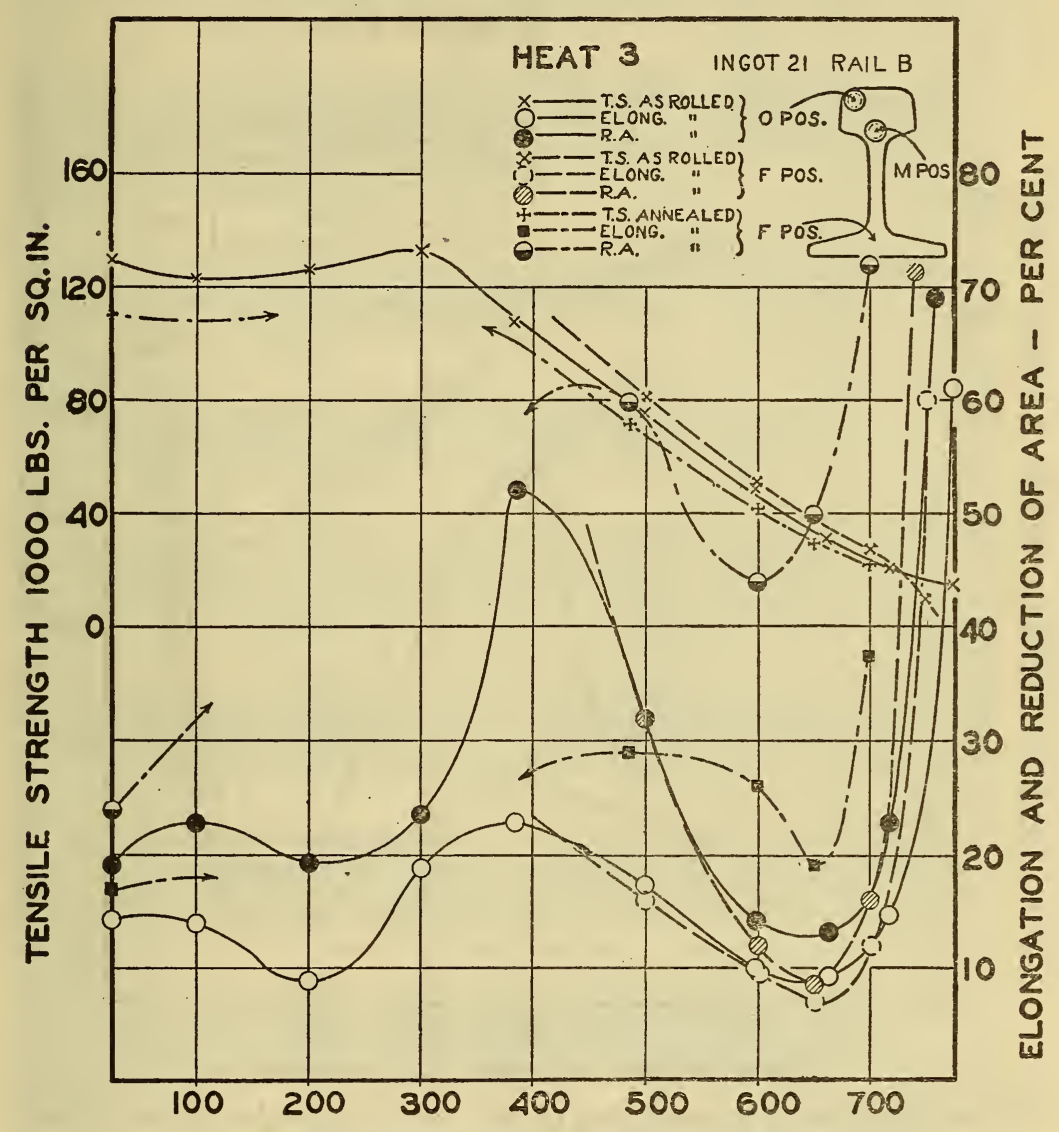

TEMPERATURE - DEGREES CENTIGRADE

FIGURE 3.-Results of tensile tests of specimens from head and base of $B$ rail, ingot 21, heat 3

marked and unexpected inversion occurred. With further increase in temperature above $400^{\circ} \mathrm{C}$. there is a marked decrease in ductility to a minimum value at approximately $650^{\circ} \mathrm{C}$. The surprising fact is evident that the elongation and reduction of area of this steel at $650^{\circ} \mathrm{C}$. is slightly less than at normal atmospheric temperatures. With increase in temperature above $650^{\circ} \mathrm{C}$. the ductility increases very rapidly to high values as the temperatures of test approach the temperature of the $A_{1}$ transformation at about $725^{\circ} \mathrm{C}$.

A photograph of a series of test bars after test is shown in Figure 4. The brittle nature of the fracture is evident. 
This temperature range, from $600^{\circ}$ to $700^{\circ} \mathrm{C}$., in which this rail steel showed such surprisingly low ductility, has been termed the "secondary brittle range" and the phenomenon "secondary brittleness." It will be referred to as such throughout this report.

Tests of specimens from the base of the rail ("F" position) were made to determine whether the phenomenon of secondary brittleness

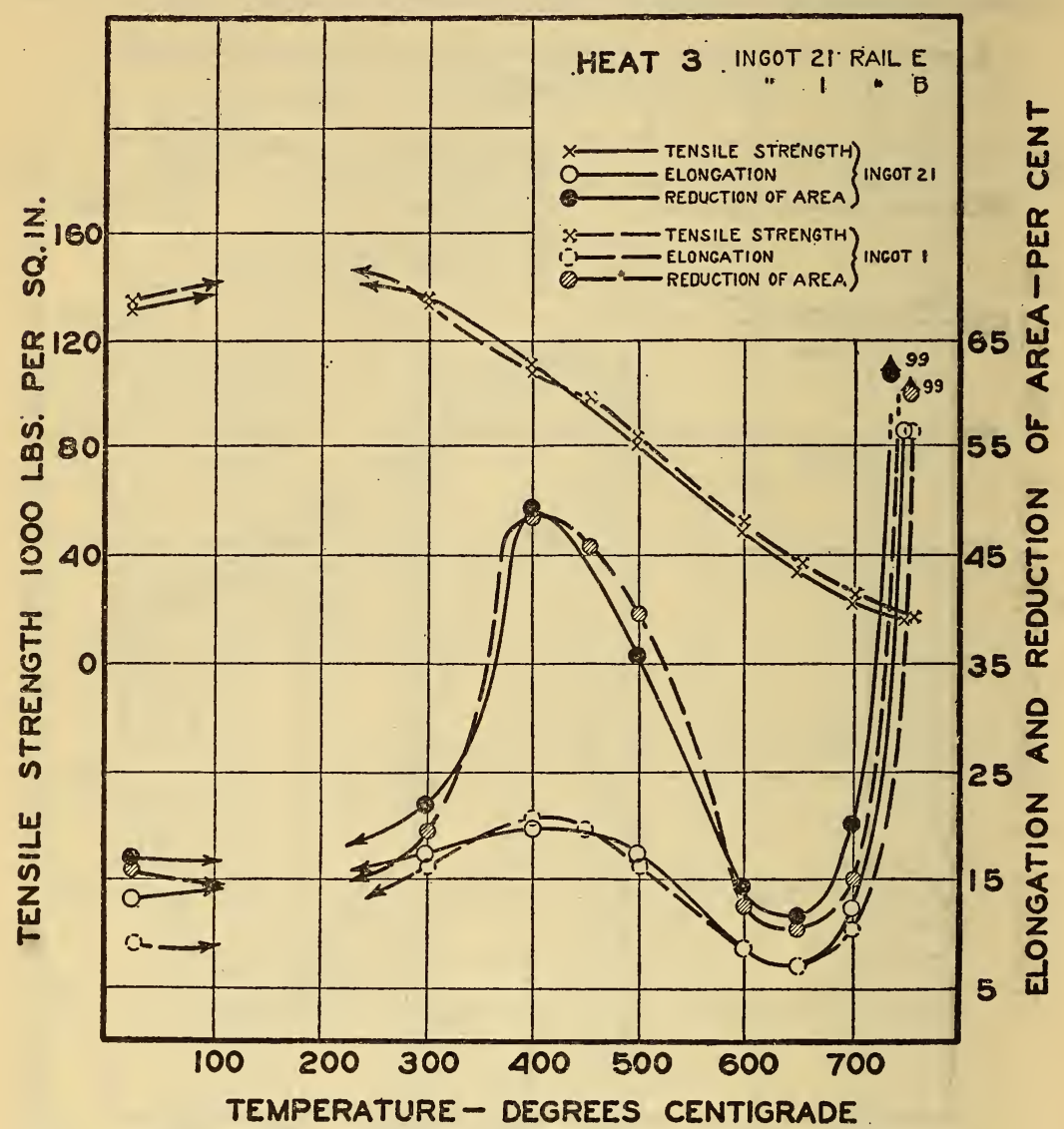

FigURE 5.-Results of tensile tests of specimens from head of $E$ rail of ingot 21 and head of $B$ rail of ingot 1 of heat 3

noted was common to the rail as a whole or possibly to an unusual segregation or allied phenomenon. The close agreement of results of tests of specimens from the base of the rail indicates quite definitely that the secondary brittleness is a property of the rail as a whole.

Similar tests were made on specimens from " $\mathrm{O}$ " position of the $\mathrm{E}$ rail of the same ingot and from the same position of the B rail of another ingot from the same heat. The results of these two series are given in Figure 5. The close agreement of the results obtained indicates quite definitely that the secondary brittleness is common to rails from near the top (B rail) and bottom ( $\mathrm{E}$ rail) of an individual ingot and also that it is common to different ingots from the same heat. Secondary brittleness appears, therefore, to be a property of the heat. 


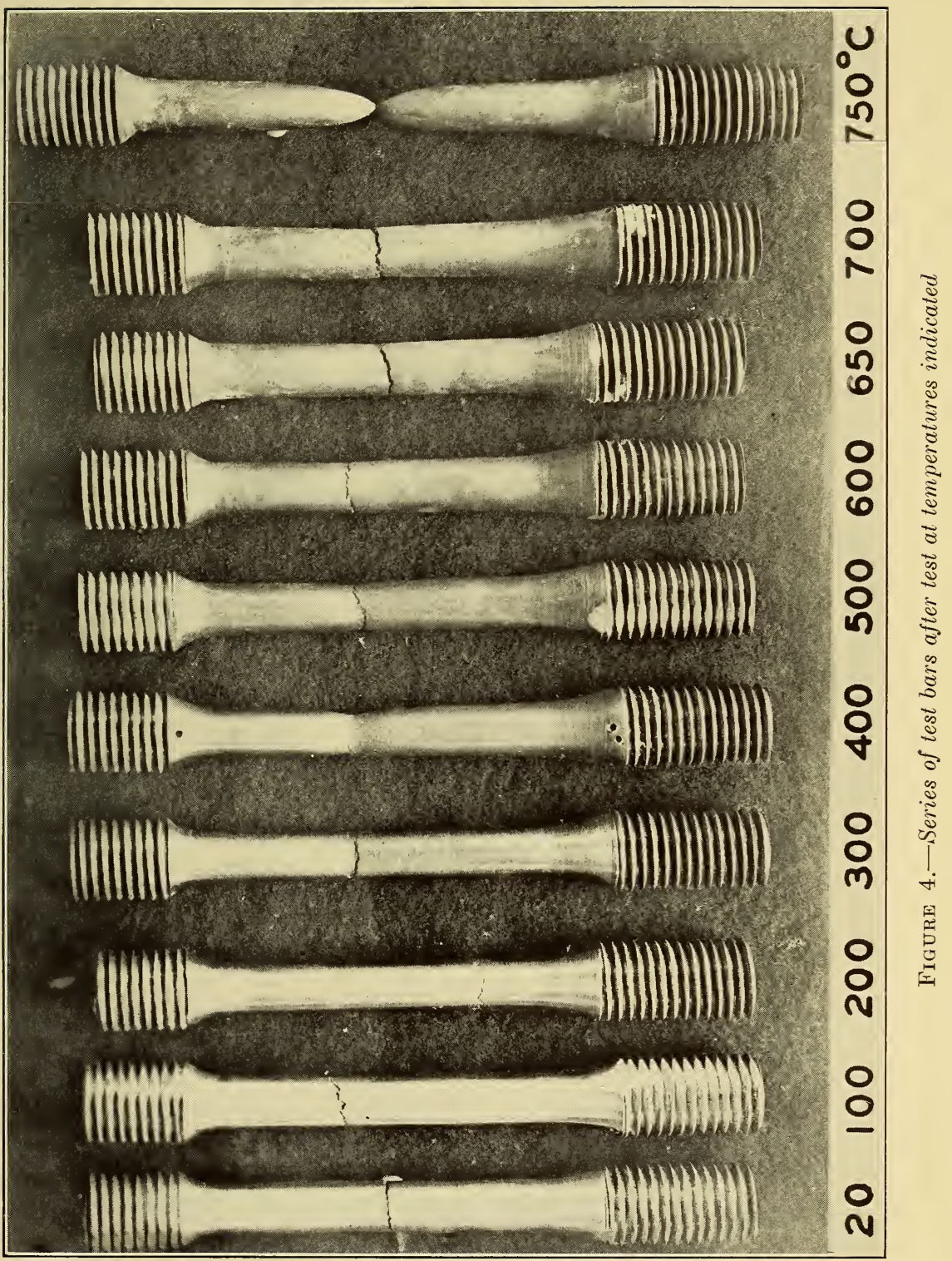


This particular heat of steel (heat No. 3) happened to be a special heat which had been killed with aluminum and poured into ingot molds with large end up and hot tops. Tests were, therefore, made on specimens from a rail known to have been made under "standard" conditions. (Heat No. 11.)

The results of these tests are given in Figure 6 . Secondary brittleness was evident in this material, but to an appreciably less marked degree. It is also noteworthy that the temperature range of secondary brittleness of this heat extends from approximately $400^{\circ}$ to $600^{\circ} \mathrm{C}$. with a minimum value of ductility at approximately $500^{\circ} \mathrm{C}$. In heat No. 3 the range of marked secondary brittleness was from approximately $500^{\circ}$ to $725^{\circ} \mathrm{C}$., with a minimum ductility occurring

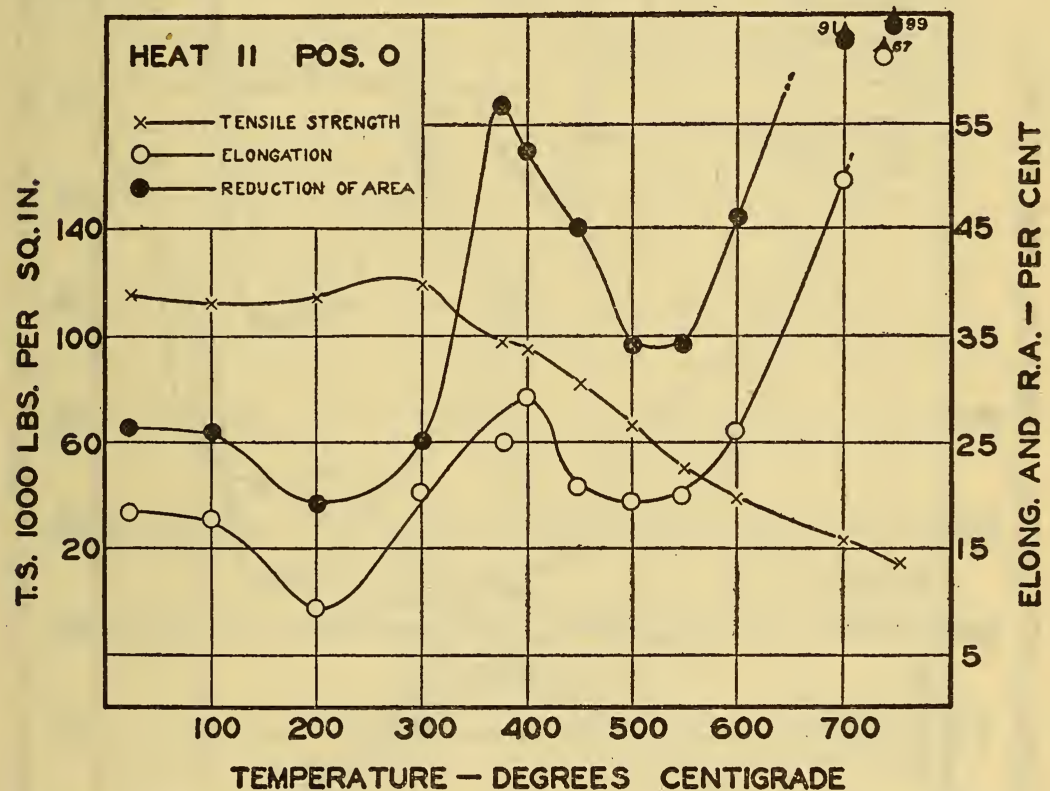

FIgURE 6.-Results of tensile tests of specimens from head of $B$ rail of heat 11

at approximately $650^{\circ} \mathrm{C}$., which is appreciably higher than indicated by heat No. 11.

Tensile tests were then made on steel from another "standard" heat rolled by another steel company. The results of the tests of specimens from both the $\mathrm{O}$ and $\mathrm{M}$ position of a $\mathrm{B}$ rail from this heat, No. 21, are given in Figure 7. Secondary brittleness is indicated in this heat, but to a relatively very minor extent. The elongation values show only a very slight drop in the secondary brittle range, and the reduction of area values while indicating a definite decrease are at all temperatures within the range appreciably higher than the corresponding values found for the other heats.

The close agreement of the values shown by the two sets of specimens taken, respectively, from the $\mathrm{O}$ and $\mathrm{M}$ position further confirm the previous indications that secondary brittleness is a property inherent to the steel. 


\section{DISCUSSION OF PRELIMINARY RESULTS}

The three steels tested were from three distinct heats of rail steel made at different times and under different conditions. Heat No. 3 was a speciäl experimental heat which was killed with aluminum and poured in big-end-up ingot molds with hot tops. Heat No. 11 was made under standard conditions at the same plant and met the same specification requirements of the Canadian Pacific Railroad. Heat

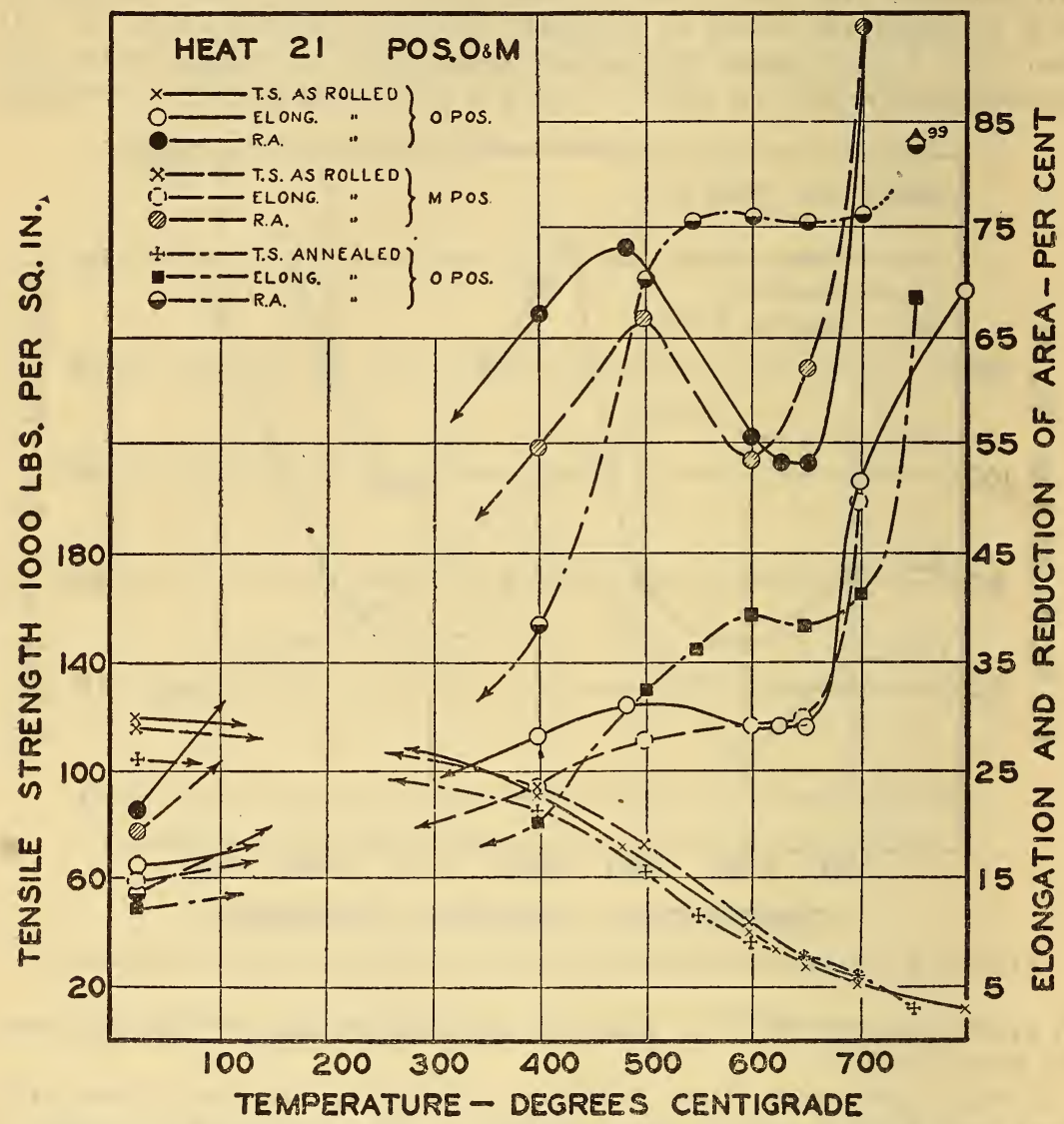

FIgURE 7.-Results of tensile tests of specimens from the $O$ and $M$ positions of a $B$ rail from heal 21

No. 21 was also made under standard conditions, but at the Carnegie Steel Co., and met the specification requirements of the Baltimore \& Ohio Railroad. The specifications of both of the above railroads are essentially the same as those adopted by the American Railway Engineering Association.

Comparison of the chemical compositions (Table 1) shows that the steels were essentially the same chemically, and all are within specification requirements. Spectroscopic analyses indicated that traces of nickel and chromium were also present, but in equal amounts in all three heats. Arsenic was determined chemically and found to be 0.01 per sent in all three steels. 
It is evident, however, that there is a marked difference in the tensile properties of the three heats at elevated temperatures, the cause of which was not apparent.

The use of aluminum to kill the steel in heat No. 3 was apparently not responsible for the marked secondary low ductility because no aluminum was used in heat No. 11, which showed a similar, although less marked, secondary brittle range. It is not known whether aluminum was added to heat No. 21.

In order to determine whether the low ductility noted at elevated temperatures was peculiar to rail steels, tests were made on bar stock

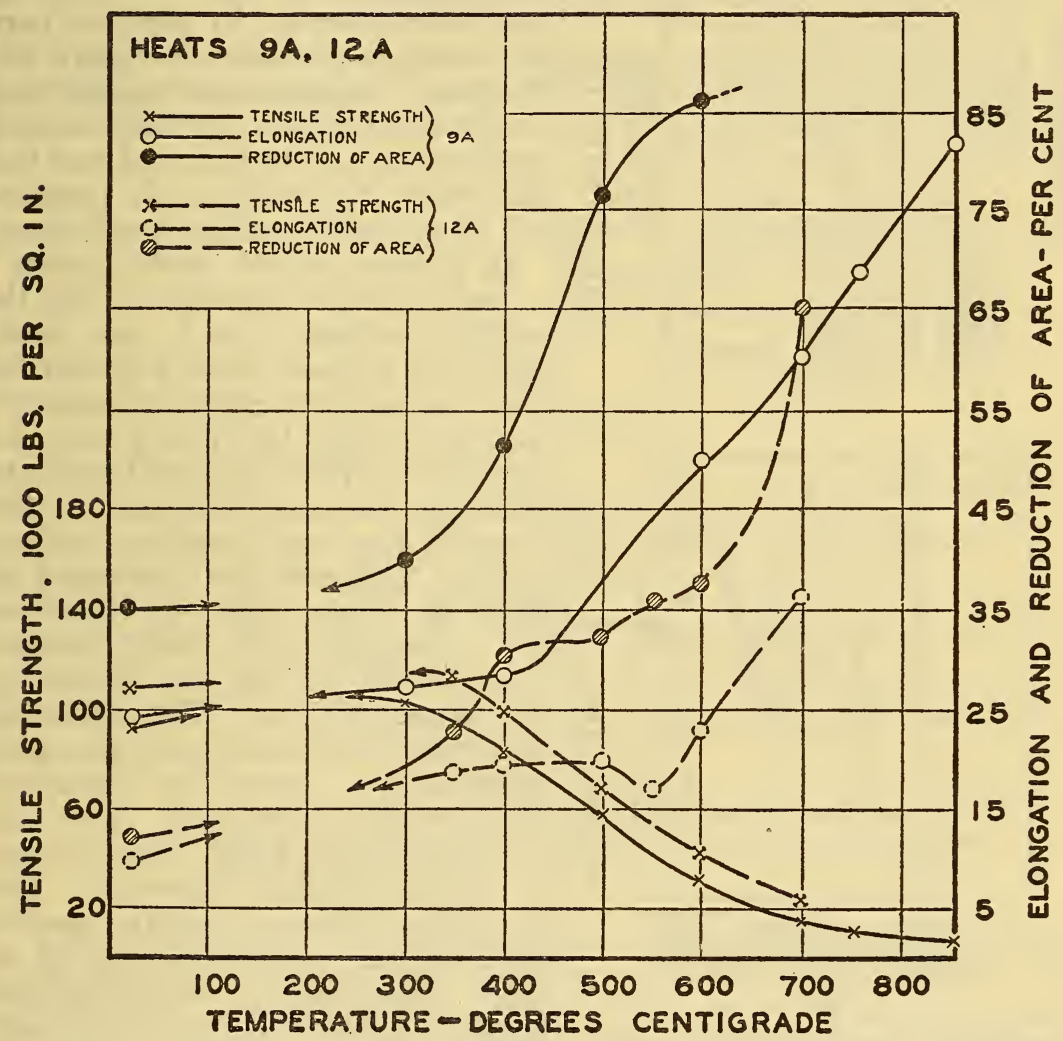

FIGURE 8.-Results of tensile tests of bar stock of approximately rail steel composition (heat 9A) and a 0.98 per cent C steel (heat 12A)

of an approximately similar composition. The history of this heat, No. $9 \mathrm{~A}$, is not known. Its composition is given in Table 1 . The results of tests of specimens from this steel are given in Figure 8. No evidence of a secondary brittle range was found. The elongation and reduction of area values increased rapidly and continuously with increasing temperature above $400^{\circ} \mathrm{C}$.

A search of the literature showed that the tensile properties of steels in the temperature range $500^{\circ}$ to $700^{\circ} \mathrm{C}$ had not been studied very intensively. A review of the data of other investigators, in the light of the present results, brought out the fact that the secondary 
brittle range had been indicated and briefly referred to, but its possible importance had not been either appreciated or emphasized.

Welter ${ }^{5}$ has reported on tensile properties of carbon steels with various manganese contents at temperatures up to $500^{\circ} \mathrm{C}$. His
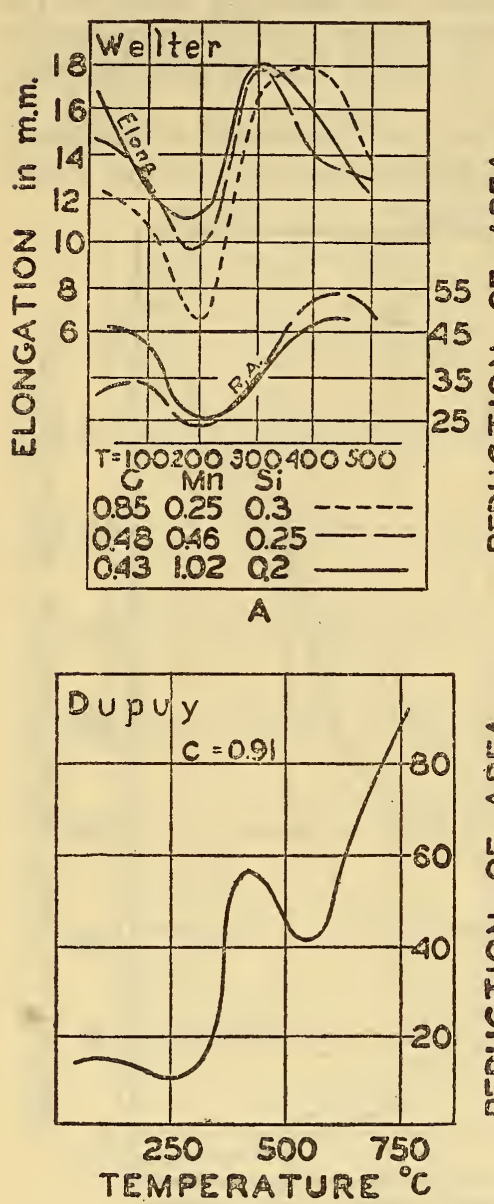

8

Figure 9.-Results of tensile tests of other investigators $A$, Welter. $B$, Dupuy. elongation and reduction of area curves indicate in several instances a distinct decrease in ductility with increase in temperature above $300^{\circ}$ to $400^{\circ} \mathrm{C}$., as is evident in Figure $9 a$.

Dupuy ${ }^{6}$ called attention to a similar inversion in the reduction of area curves for a 0.91 per cent carbon steel as is shown in Figure $9 b$. There are indications of similar inversions in his data for the low and medium carbon steels tested, but the data were not sufficiently consistent to support definite conclusions.

In a more recent investigation ${ }^{7}$ a very definite secondary brittle range is indicated in a cast steel. Since the present work was started evidence has been given by Inokuty ${ }^{8}$ that secondary brittleness may be present in Armco iron although its presence was not indicated in his tests of low and medium carbon steels. This was also indicated in tests of Armco iron by Rawdon and Berglund. ${ }^{9}$ A very definite confirmation of this was found by the present writers. The results of these tests of Armco iron at elevated temperature, found by Inokuty, Rawdon and Berglund, and the present writers, have been plotted to the same scale in Figure 10. The very close agreement of the results obtained on a similar material in three independent investigations made at different times is unusually positive evidence of the existence of the phenomenon. It is also evident from the curves in Figure 10 that what has been termed secondary brittleness is distinct from the wellknown phenomenon of hot-shortness in relatively pure irons which occurs at approximately $900^{\circ} \mathrm{C}$. This hot-short range is shown in Figure 10 in the curves based on Inokuty's data.

s Welter, G., Elastizität u. Festigheit von Specialstählen bei hohen Temperaturen-Forschungsarbeiten a. d. Gebeite d. Ingeneiurswesen, 230, p. 1; 1921.

6 See footnote 2, p. 550.

7 Pioneering in Science, Crane Co. Circular No. 163, An Investigation in the Effect of High Temperature on Metals, p. 26.

8 Inokuty, T., Tensile Strength of Steels at High Temperatures, Sci. Repts. of Tohoku Imperial Univ.; July, 1928.

0 Rawdon, H. S., and Berglund, T., Unusual Features in the Microstructure of Ferrite, B. S. Sci. Paper No. 571. p. 698, Figure 3(b). 
The data obtained on rail steels, bar stock of rail steel composition, Armco iron, and the similar results reported by other investigators all indicate that secondary brittleness may be present in many steels under certain as yet unknown conditions. The desirability of a more extended study of the phenomenon was thus emphasized especially in view of its possible relation to the presence of internal cracks-shatter cracks-in rail and other steels as discussed fully later in this report.

\section{TENSILE PROPERTIES OF SEVERAL RAIL STEELS AT ELEVATED TEMPERATURES}

\section{TRANSVERSE FISSURED RAILS}

For the studies of endurance properties previously referred to two transverse fissured rails, ${ }^{10}$ and a rail which had been subjected to the same identical service in track as one of them, had been obtained.

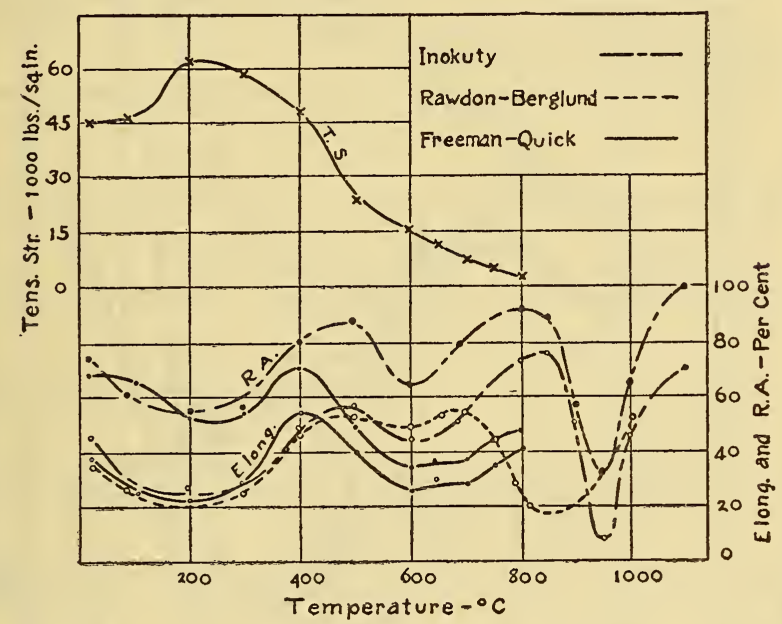

FIgURE 10.-Results of tensile tests at elevated temperatures of Armco iron by Inokuty, Rawdon and Berglund, Freeman and Quick

One of these rails (PO) failed in track on the Pennsylvania Railroad after only about five months' service, and it had been found at their laboratories ${ }^{11}$ and also at the Bureau of Standards to contain numerous shatter cracks and incipient fissures. The rail (CN1) which failed in track on the Canadian National Railway after over eight years' service was also shown to have contained shatter cracks and incipient fissures. In the companion rail (CN2), which had been subjected to identical service and had not failed, no shatter cracks were found.

Tensile tests were made on specimens taken from the $\mathrm{O}$ position in the heads of these rails. The results for rail PO are given in Figure 11 , and for rails $\mathrm{CN} 1$ and $\mathrm{CN} 2$ in Figure 12 . It is evident that secondary brittleness is present in all cases. It is particularly marked in rail PO (fig. 11) which showed marked internal shattering.

10 These are rails PO from the Pennsylvania R. R. and CN1 and CN2 from the Canadian National Ry. See footnote 4, p. 551

11 Cushing, W. C., Rail and Wheel, Proc. Am. Rwy. Eng. Assoc., 30, No. 315, Pt. 1; 1929. See particularly p. 268. 
The elongation of about 9 per cent shown by this steel at the minimum of the secondary brittle range which occurs at about $550^{\circ} \mathrm{C}$. is practically the same as at normal temperatures. Similarly, the reduction of area of about 14 per cent at this temperature is practically the same as exists at normal temperatures. The tensile strength, however, is only about 47 per cent of the value obtained at normal temperature.

The degree of secondary brittleness in rails $\mathrm{CN} 1$ and $\mathrm{CN} 2$ (fig. 12) is not so marked as in rail PO, although appreciable. There is a slight

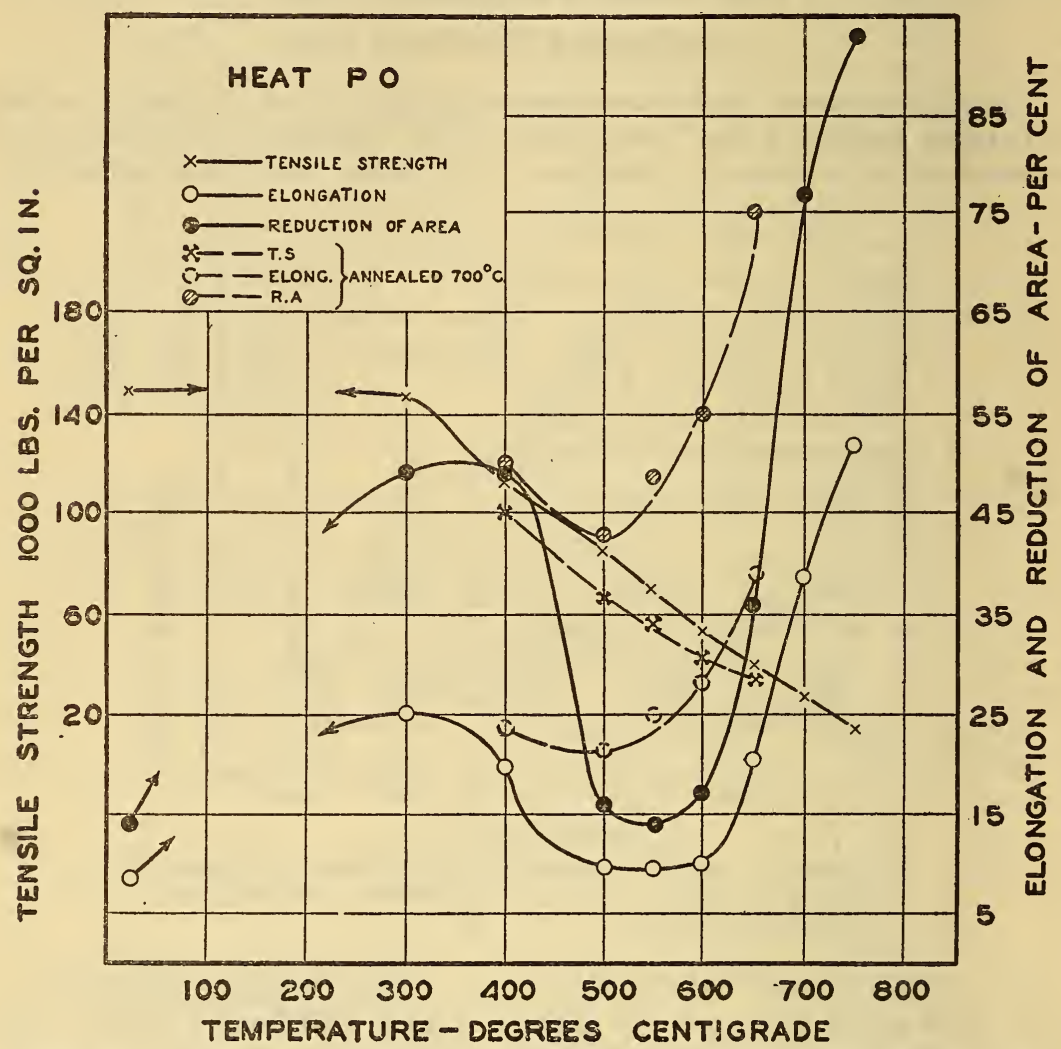

FIgURE 11.-Results of tensile tests at elevated temperatures of specimens from the $O$ position of transversed fissured rail $(P O)$ from Pennsylvania Railroad

difference in the tensile properties of the two steels, the steel from the fissured rail having an appreciably lower elongation and reduction of area and slightly higher tensile strength at all temperatures.

\section{HEAT-TREATED RAILS}

At the request of the Joint Rail Manufacturers Technical Committee and the Rail Committee of the American Railway Engineering Association endurance tests have been made ${ }^{12}$ of special heattreated rails. These rails were made and heat treated in accordance

12 To be reported in B. S. Jour. Research and Bull. A. R. E. A. 
with the Kenney process. ${ }^{13}$ The rails from one heat (H1) were quenched in water for 30 seconds. The rails from the other heat (H2) were quenched for 15 seconds. In each case immediately after quenching the rails were transferred to a furnace preheated to $950^{\circ} \mathrm{F}$. and were held at this temperature for one and one-half hours and were then allowed to cool in air. Tensile tests were made on speci-

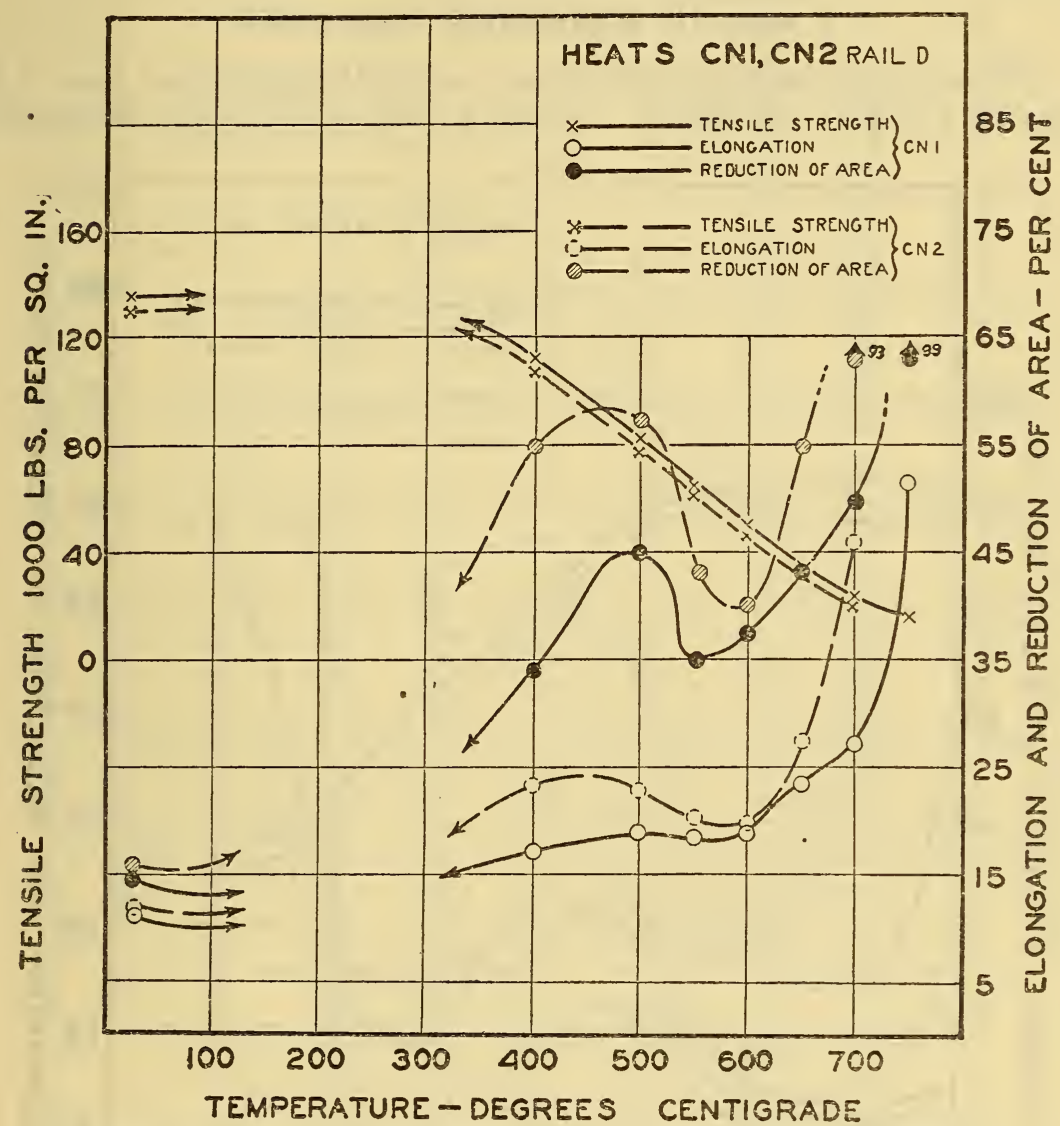

FIGURE 12.-Results of tensile tests at elevated temperatures of specimens from a transverse fissured rail (CNl) and, an adjoining rail in track which had not failed under the same service conditions on the Canadian National Railway

mens from the " $\mathrm{O}$ " position of the heads of a " $\mathrm{C}$ " rail representative of each of the two conditions of heat treatments. The results are given in Figure 13. It is evident that there is a marked difference in the tensile properties of the two steels at all temperatures of test. It has been shown previously that different heats of rail steel may have marked difference in secondary brittleness. Since these two series were from different heats it is therefore impossible to state

${ }^{13}$ E. F. Kenney, Heat Treatment of Steel, U. S. Patent No. 1619025, and Heat Treatment of Railway Rails. Reissue No. 17240 . 
what influence the difference in heat treatment may have had on the secondary brittleness. It is of interest, however, to find that such a marked difference may exist, especially in reduction of area.

Similar tests were also made on a " $\mathrm{C}$ " rail from another heat (H3) made by the same company which was made in the usual manner. The results of these tests are also given in Figure 14.

\section{MEDIUM MANGANESE RAIL STEEL}

The so-called medium manganese rail steels containing from 1.2 to 1.70 per cent manganese are being used in increasing amounts.

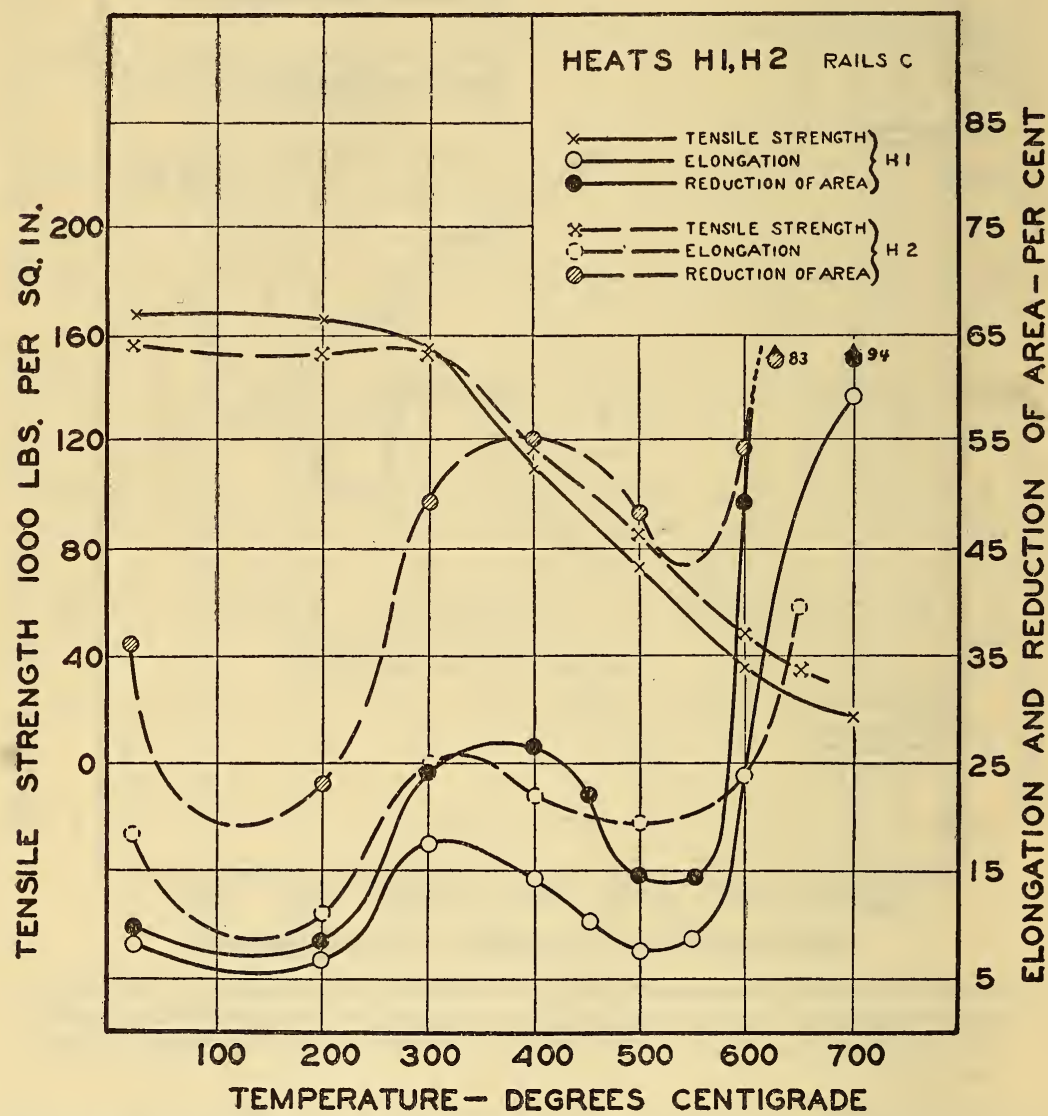

FigURe 13.-Results of tensile tests at elevated temperature of heat-treated rail steels

$H 1 C$, rail quenched 30 seconds in water.

$H 2 C$, rail quenched 15 seconds in water.

Both rails after quenching held $11 / 2$ hours in furnace at $950^{\circ} \mathrm{F}$., then air cooled.

Tensile tests have been made at elevated temperatures using specimens from the "O" position of two D rails containing about 1.5 $\mathrm{Mn}$ (M1D) and 1.20 (M2D), respectively, the compositions of which are given in Table 1. The results of the tensile tests are given in Figure 15. There is relatively very slight difference in the tensile 
properties of these two steels at the various temperatures of test. Secondary brittleness is very marked in both instances. The elongation and reduction of area values at the minimum of the secondary brittle range, which occurs at about $550^{\circ} \mathrm{C}$., are appreciably lower

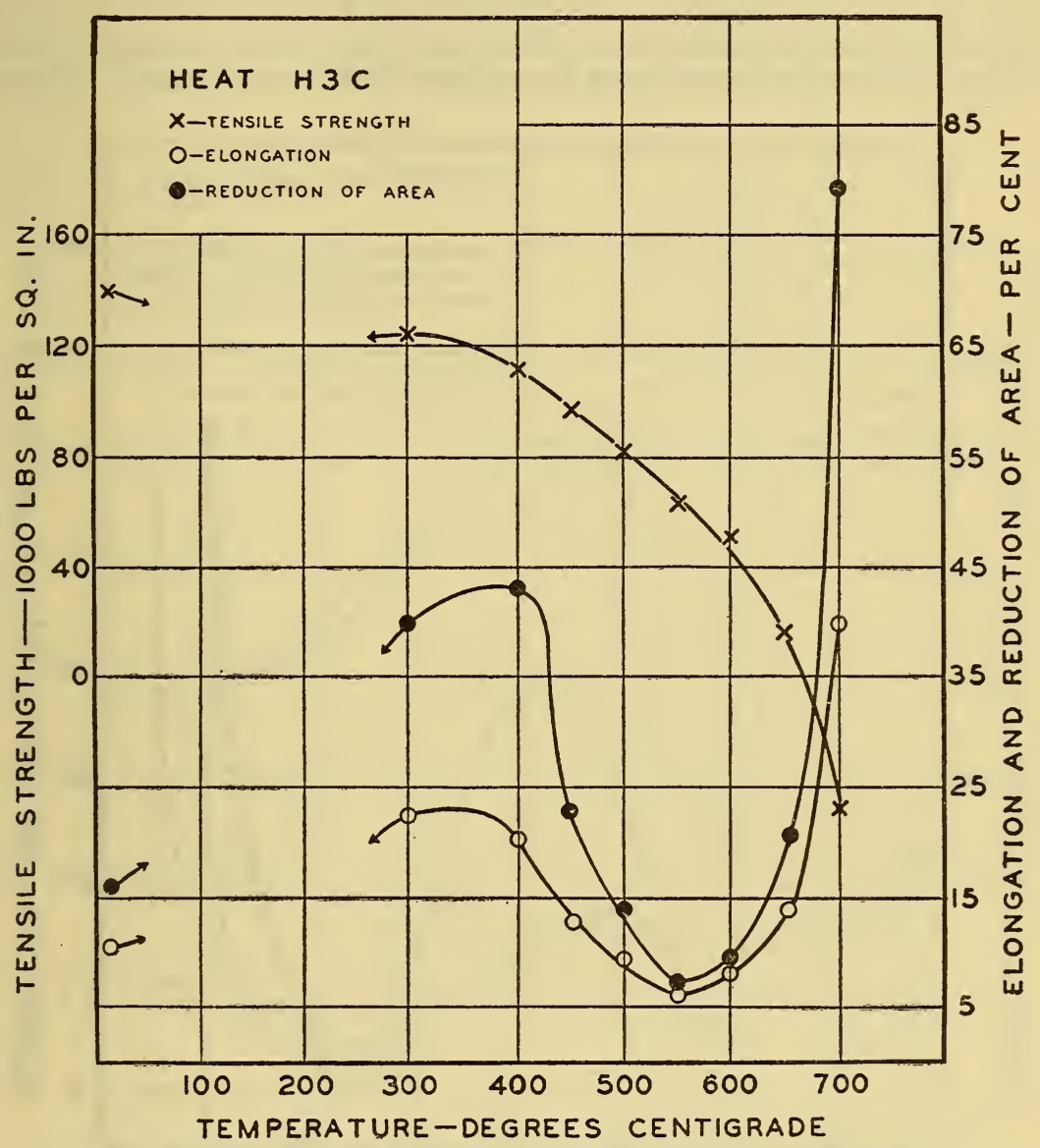

FIGURE 14.-Results of tensile tests at elevated temperature. Heat HS, $C$ rail

than at normal $\left(20^{\circ}\right.$ C.) temperatures. The tensile strength at $500^{\circ} \mathrm{C}$. is about 50 per cent of the value at normal temperatures.

\section{MANGANESE MOLYBDENUM RAIL}

At the request of G. N. Eaton, of the Molybdenum Corporation of America, tensile tests were made at elevated temperatures of a special manganese-molybdenum rail steel, the composition of which is given in Table 1. The results of this series of tests are given in Figure 16. The test specimens were taken from the "O" position as in the other series. Secondary brittleness is also present in this material, a minimum elongation and reduction of area occurring at approximately $600^{\circ} \mathrm{C}$. It is of interest to note the relatively high values of ductility, especially of reduction of area indicated by this material at normal 
$\left(20^{\circ}\right.$ C.) temperatures. The total number of tests made, one at each temperature, are rather few, so that definite conclusions can not be drawn, and the data, therefore, must be considered as tentative only.

\section{RAIL STEEL POURED AT HIGH TEMPERATURES}

In a previous investigation, ${ }^{14}$ data are given on the properties of a rail steel known to have been excessively hot when poured. A sec-

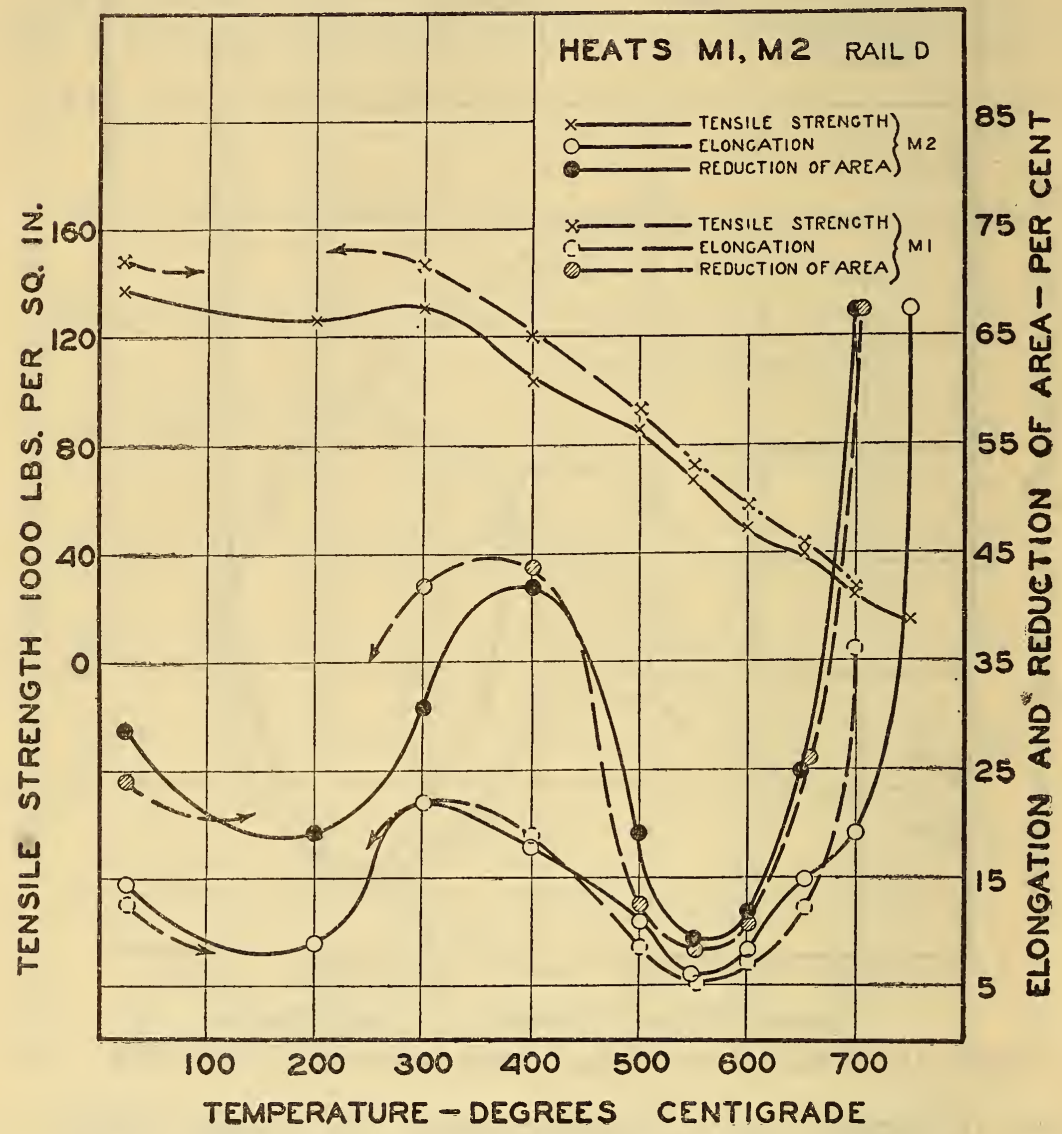

FIGURE 15.-Results of tensile tests at elevated temperature of medium manganese rail steels

$M 1 D, 1.5$ per cent $\mathrm{Mn}$; $M 2 D, 1.2$ per cent $\mathrm{Mn}$.

tion of the $B$ rail, the properties of which had been previously determined, was available and afforded the opportunity of determining at least qualitatively whether pouring temperature had any marked influence on secondary brittleness. Results of elevated temperature tests on specimens from the "O" position of this rail are given in Figure 17. Apparently a high pouring temperature has by itself no marked influence on the degree of secondary brittleness in a rail steel. 


\section{STEEL FROM A RAIL FROM A REHEATED BLOOM}

In a report of studies by the Pennsylvania Railroad, ${ }^{15}$ data are given on the relative service of direct-rolled rails and rails from blooms taken from the same heats and which had been allowed to become cold and were then reheated for rolling into rail. The service data on these rails taken over a period of several years showed that rails from reheated blooms gave better service. Transverse fissure failures were

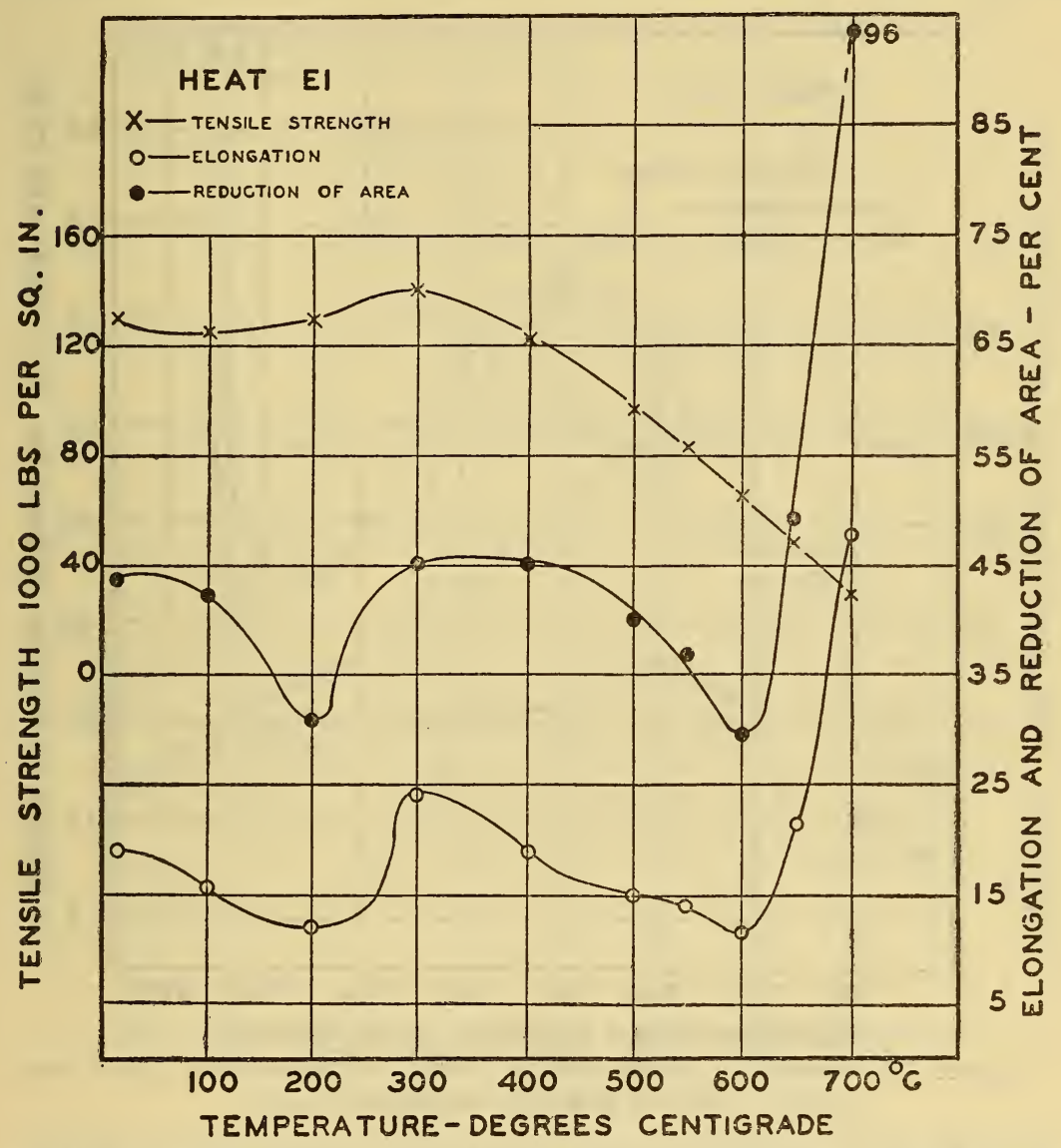

FIgURE 16.-Results of tensile tests at elevated temperature of a manganesemolybdenum rail steel, heat E1

less in these rails than in direct-rolled rail. At the suggestion of Dr. M. E. McDonnell, chief chemist of the Pennsylvania Railroad, tensile tests were made at elevated temperatures on specimens from two rails from the same heat, for the purpose of determining whether reheating of the bloom had caused any appreciable differences in the degree of secondary brittleness. One of these rails (PH1) was known to be from a reheated bloom and the other $(\mathrm{PH} 2)$ from a direct-rolled bloom. The results of these tests are given in Figure 18. It should be noted 
that both rails were from the same heat, the only known difference being the reheating of the bloom of rail PH1. Secondary brittleness is present in a relatively moderate degree in both instances. In previous work reported in this paper, it was indicated that secondary brittleness was apparently a property of the heat. Since these two rails were from the same heat it is a reasonable assumption that the difference in secondary brittleness is a result of the reheating of the bloom. Whether it is directly the result of reheating the bloom or

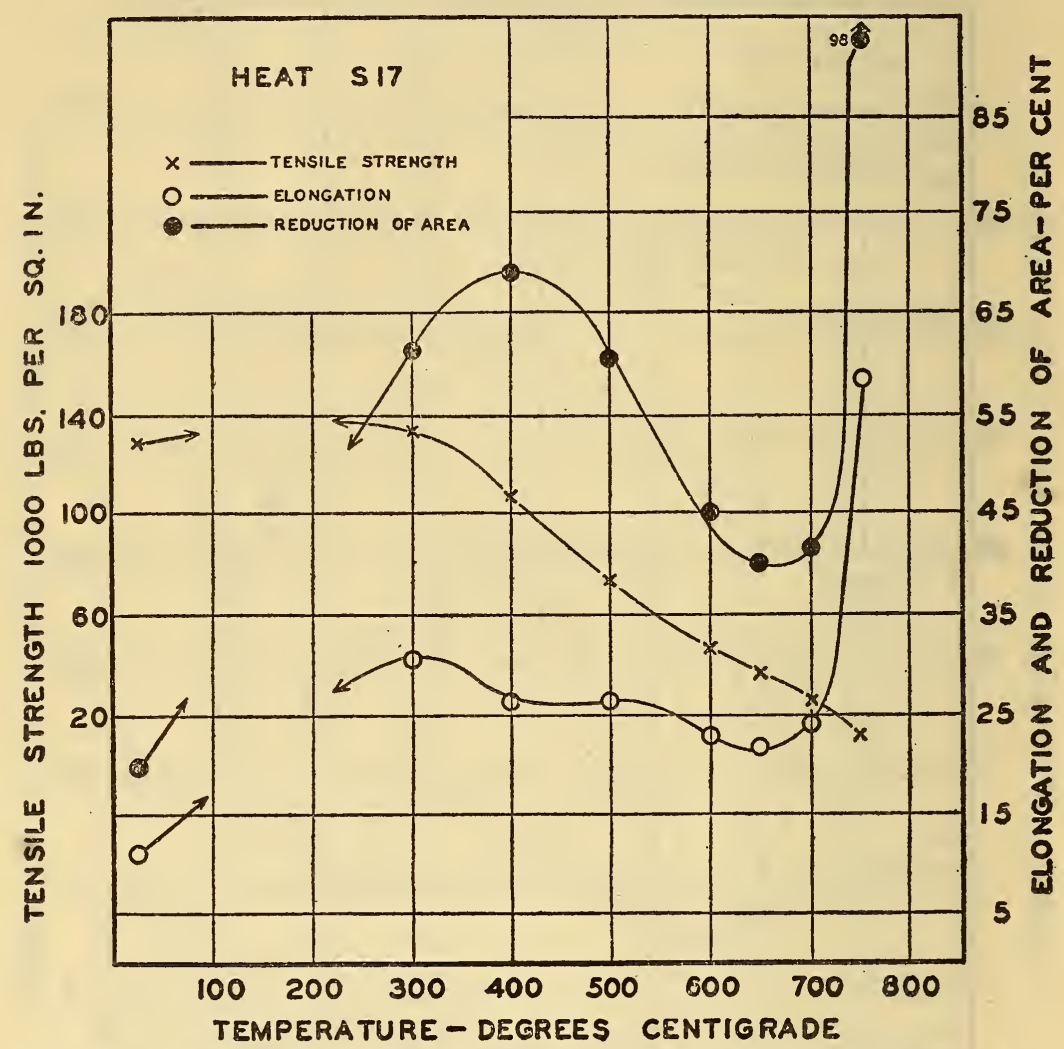

FIGURE 17.-Results of tensile tests at elevated temperature of a rail steel poured at a high temperature

indirectly the result of some other slight but unknown change in process, such as finishing temperature, or rate of cooling can not be stated. This is particularly noteworthy because, as shown later in this report, annealing even below the critical range has an appreciable influence on secondary brittleness. It so happens that none of the rails from this heat either from direct-rolled or reheated blooms, developed transverse fissures in track. Therefore, no conclusions can be drawn regarding any possible relation of secondary brittleness to the relative freedom from transverse fissues of rail from reheated blooms. 


\section{TENSILE PROPERTIES AT ELEVATED TEMPERATURES OF SOME STEELS OTHER THAN RAIL STEELS}

\section{CHROME-MOLYBDENUM TIRE STEEL}

In discussing secondary brittleness in rail steels and its possible relation to internal failures, as brought out more fully later in this report, it was pointed out by G. M. Eaton that certain heats of chrome-molybdenum locomotive tire steels were known at times,

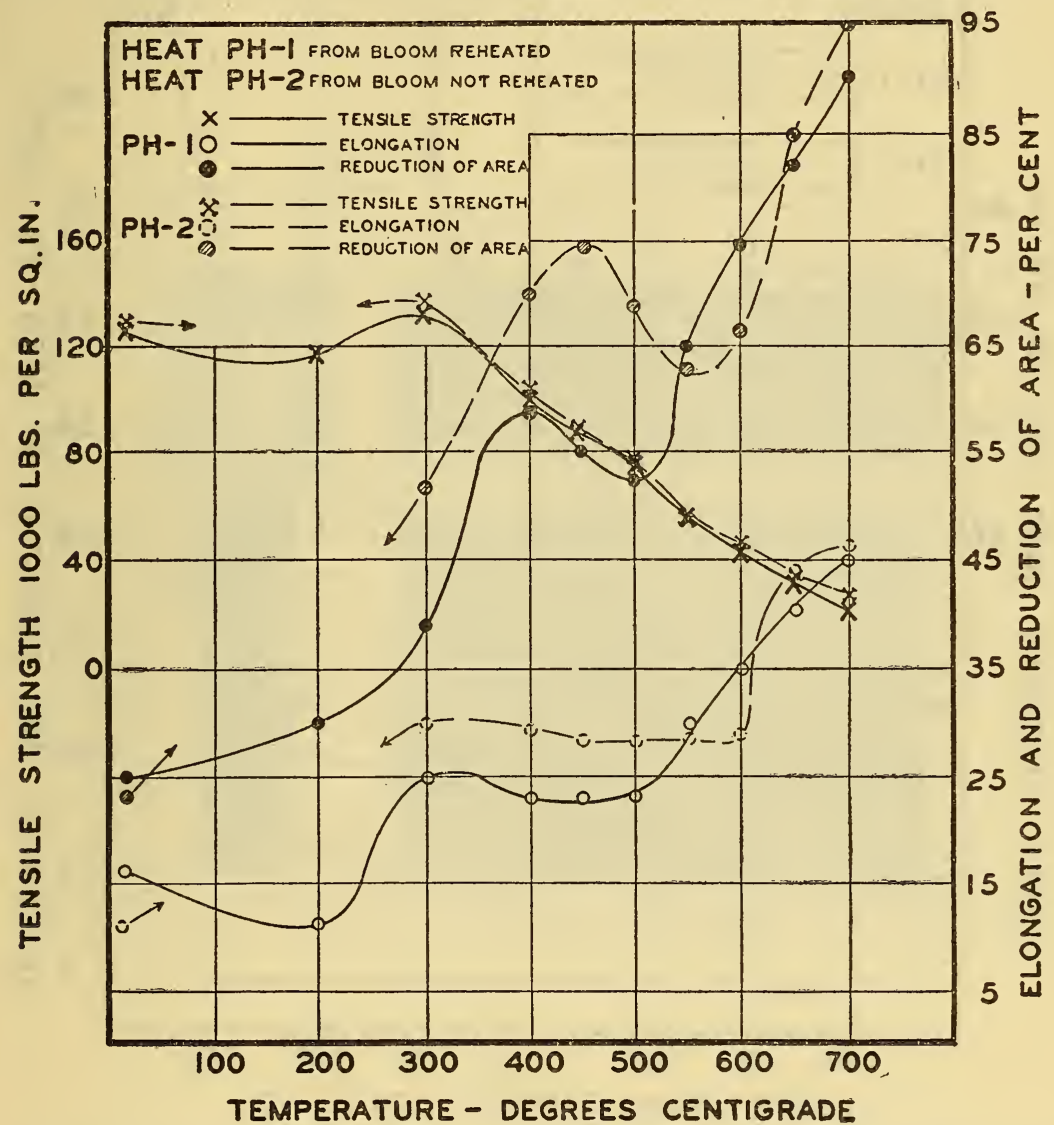

FIGURE 18.- Results of tensile tests at elevated temperature of rail steel from "direct" rolled and "reheated" blooms

PH1, reheated bloom.

$P H 2$, direct rolled bloom.

although infrequently, to develop cracks if allowed to cool in air from the forging temperature, but if caused to cool slowly by burying under mill scale after forging no such difficulty was encountered. Through the courtesy of Lawford H. Fry, metallurgical engineer, Standard Steel Works, two series of test specimens were obtained. Both series were from the same heat of steel. One series (ST1), however, was taken from a tire which was cooled in air after rolling and developed cracks. The other series (ST2) was taken from a similar tire which had been cooled slowly by burying in mill scale after rolling. 
The results of the tests are given in Figure 19. Secondary brittleness was present in each case, but there is a very marked difference in degree. The effect of the slower cooling evidently has been to decrease the magnitude of secondary brittleness. With the higher elongation and reduction of area available the steel would seem to be in a better condition to flow and so relieve any destructive thermal stresses that might be set up during cooling. Of course, the slower

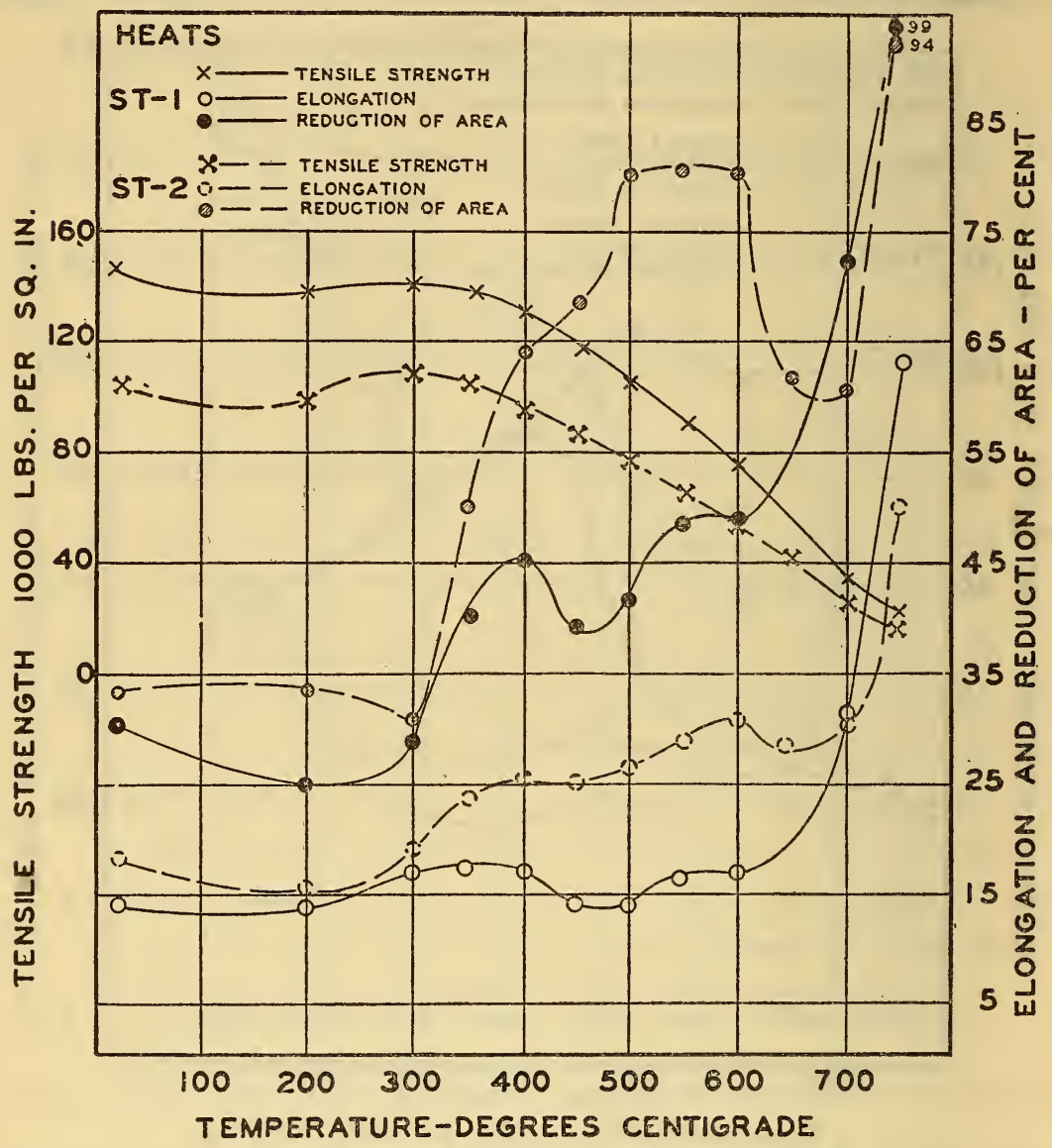

FigdRE 19.-Results of tensile tests at elevated temperatures of a chromemolybdenum tire steel

$S T 1$, tire cooled from forging temperature in air

$S T 2$, tire cooled slowly from forging temperature by burying in mill scale.

cooling would in itself greatly reduce the tendency of thermal stresses to develop and their magnitude as compared to the more rapid air cooling. The effect of the slow cooling then would seem to be a dual one; the magnitude of the stresses is reduced and at the same time the steel is apparently put into a condition less liable to failure should high stresses be developed. Sufficient data are not available to determine the relative importance of the two factors. 
B. S. Journal of Research, RPI64

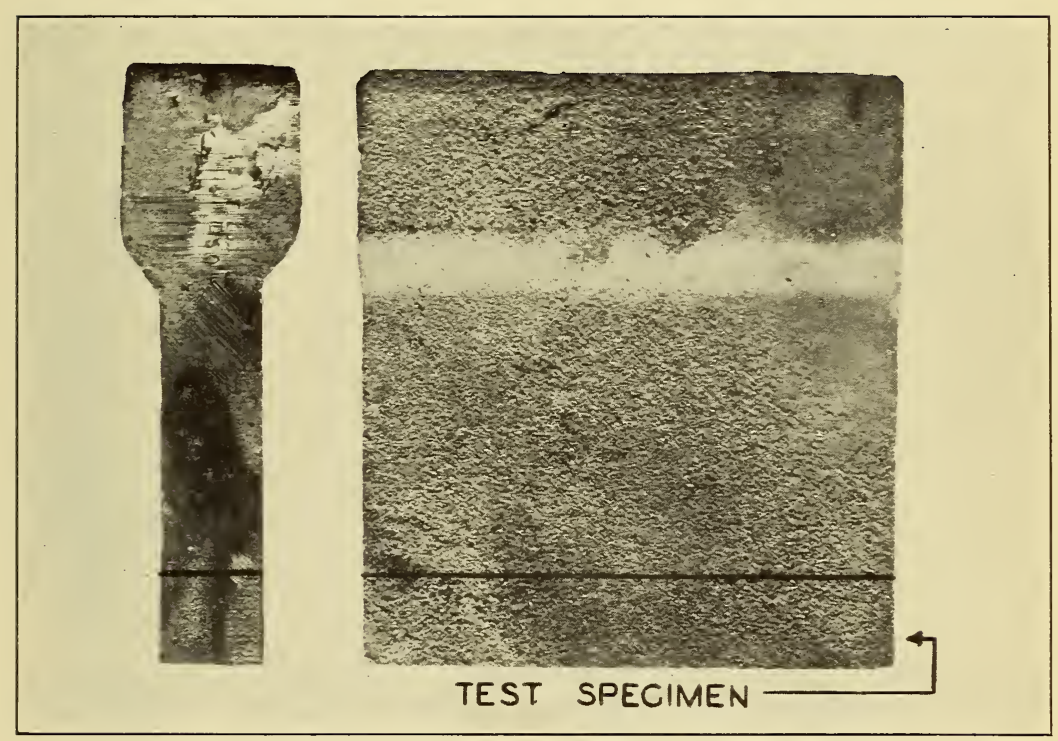

FIgURE 20.-Type of casting used for cast-steel test bars All specimens were taken from the bottom as indicated. 


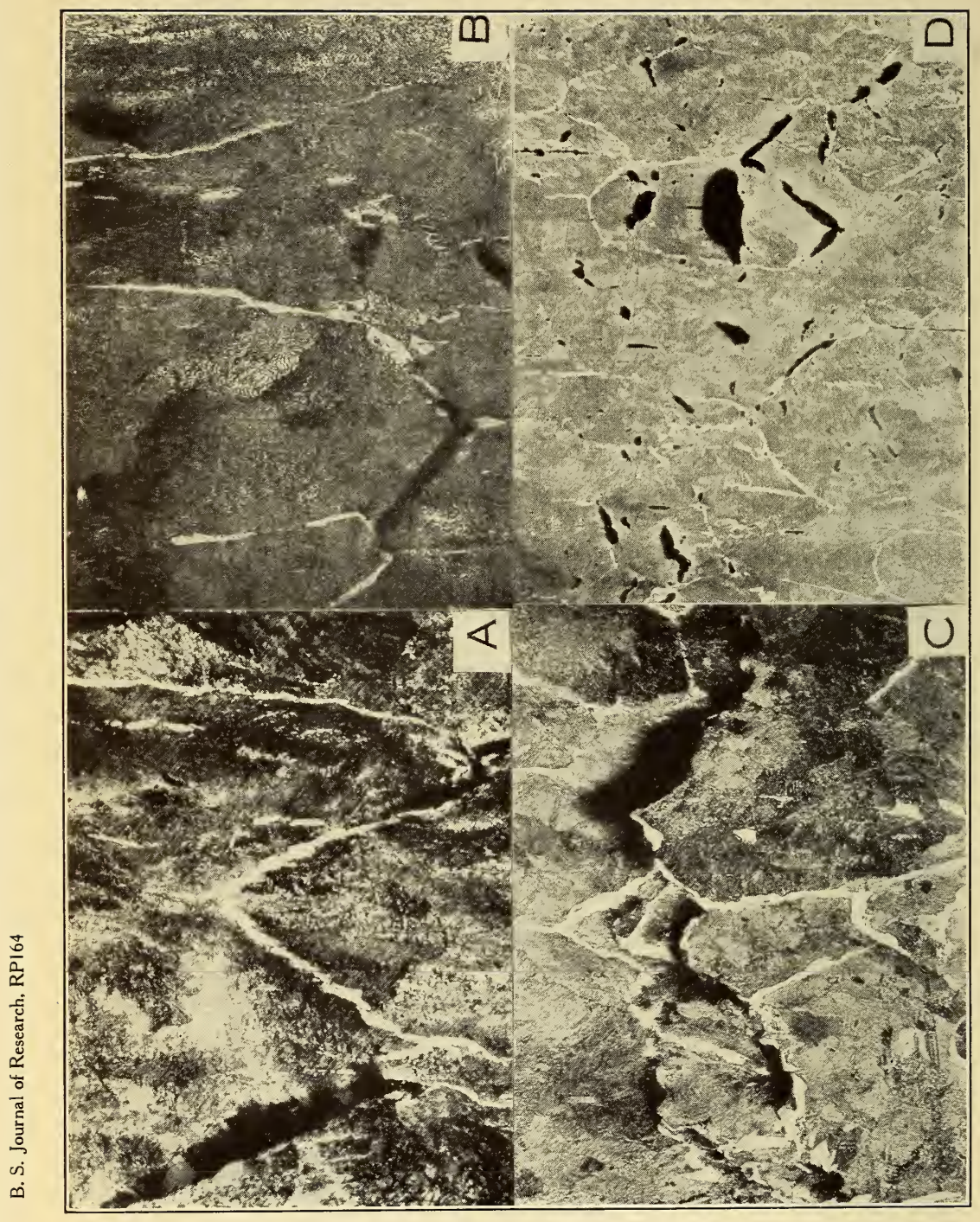

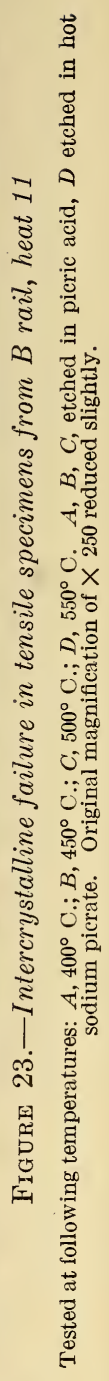




\section{ONE PER CENT CARBON STEEL}

It is known that free cementite is sometimes found in rail steels. It will be noted in Table 1 that the manganese content in the rail steels is relatively high for steels of similar carbon content. It is known that manganese tends to shift the eutectoid ratio of carbon steels in the direction of lower carbon content. It is conceivable, with the manganese-carbon ratio present in these rail steels, that under certain conditions of segregation and rate of cooling free

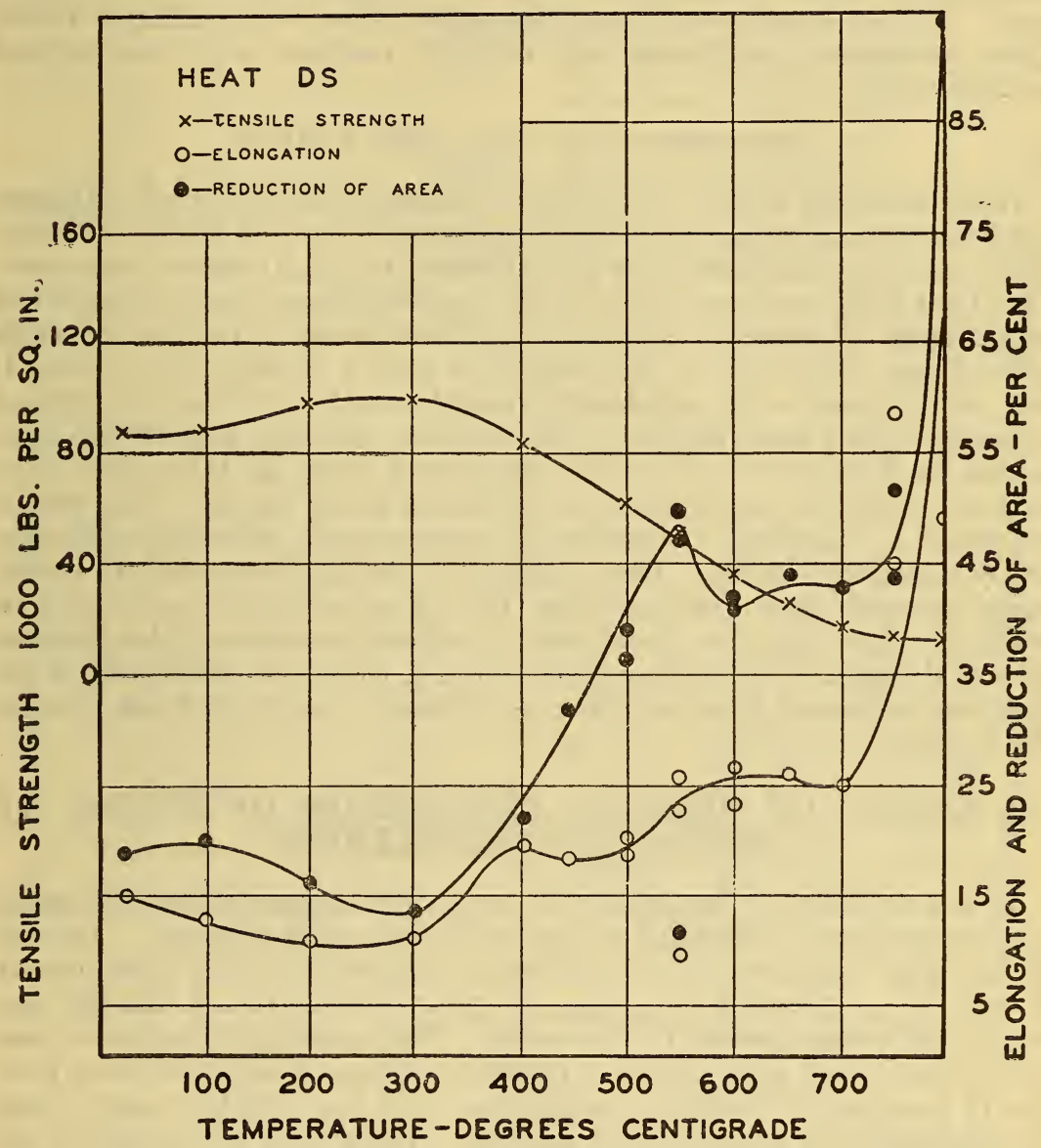

FIgURE 21.-Results of tensile tests at elevated temperatures of a 0.30 per cent carbon cast steel, heat $D S$

cementite might be present in the rail steels tested. It was thought that the secondary brittleness might be associated with its presence.

Tests were, therefore, made on a high-carbon steel (0.98 per cent C) in which free cementite was known to be present. The history of the bar from which the specimens were taken was unknown except that before cutting off the specimens the bar was annealed so as to produce free cementite at the grain boundaries.

The results of the tensile tests are included in Figure 8. Secondary brittleness is present in the high-carbon hypereutectoid steel $(0.98 \mathrm{C})$ 
to an appreciable extent. Whether the secondary brittleness is related to the presence of the free cementite, however, can not be stated, since it was found, as is shown later in the investigation, in Armco iron and steels in which free cementite is not ordinarily present, at least in appreciable amounts. The fact that secondary brittleness was found in the annealed hypereutectoid steel is of interest and is the more significant when it is realized that the steel was in a fully annealed condition for, as is shown later, it is known that full annealing tends to reduce markedly the degree of secondary brittleness. It may be inferred then that under some as yet unknown condition secondary brittleness may be quite marked in hypereutectoid carbon steels.

\section{CAST STEEL (0.30 PER CENT CARBON)}

In some earlier work ${ }^{16}$ secondary brittleness was indicated, although not commented upon, in the ductility curves of a 0.30 per cent carbon cast steel. A set of test bars was obtained of a 0.30 carbon cast steel. This heat (DS) was made in an acid electric furnace under commercial conditions. A quantity sufficient for the number of test-bar castings desired was poured from the bull ladle into a smaller ladle where it was recarburized with preheated washed metal. It was then poured into dry (core) sand molds. The test-bar castings were of the type shown in Figure 20. The test specimens were all taken from the bottom portion of the casting, as indicated in the figure. The results are given in Figure 21. Secondary brittleness is indicated to a relatively moderate degree. One specimen tested in the secondary brittle range showed very low ductility, the cause of which could not be definitely determined as being associated with cracks or blowholes or similar defects. The values obtained are, therefore, indicated in the diagram; a second specimen from a different casting did not confirm the results.

\section{EFFECT OF RATE OF APPLICATION OF STRESS ON SECONDARY BRITTLENESS}

It is a well-known fact that the values determined in tensile testing may vary appreciably with the rate of application of stress. In tests at elevated temperatures this effect is in general more pronounced. A rate of application of stress of about 0.28 inch a minute was used in all tests reported in this work. To determine the effect of rate of application of stress a series of tests were made on specimens from the $\mathrm{O}$ position of medium manganese steel rail (M2D) using a rate of application of 2.08 inches a minute. Tests were made only in the secondary brittle range. The results are given in Figure 22 . It is evident that at the higher rate of application of stress the elongation and reduction of area are slightly greater. Due to the rapid rate used it was not possible to keep the beam of the testing machine correctly balanced. Data for tensile strength values at the higher speed were, therefore, not determined.

The data show very definitely that secondary brittleness is not related, at least in any marked degree, to the speed of testing.

16 See footnote 7, p. 558. 


\section{EFFECT OF ANNEALING ON SECONDARY BRITTLENESS}

A series of preliminary tests was carried out to determine the effect of annealing on the secondary brittleness. A section of the $B$ rail of heat No. 21 on which previous tests were made (fig. 7) was annealed by heating at $1,000^{\circ} \mathrm{C}$. for six hours and cooling slowly in the furnace. Tensile tests were then made on specimens from the $\mathrm{O}$ position. The results have been plotted in Figure 7 for ease of comparison with the

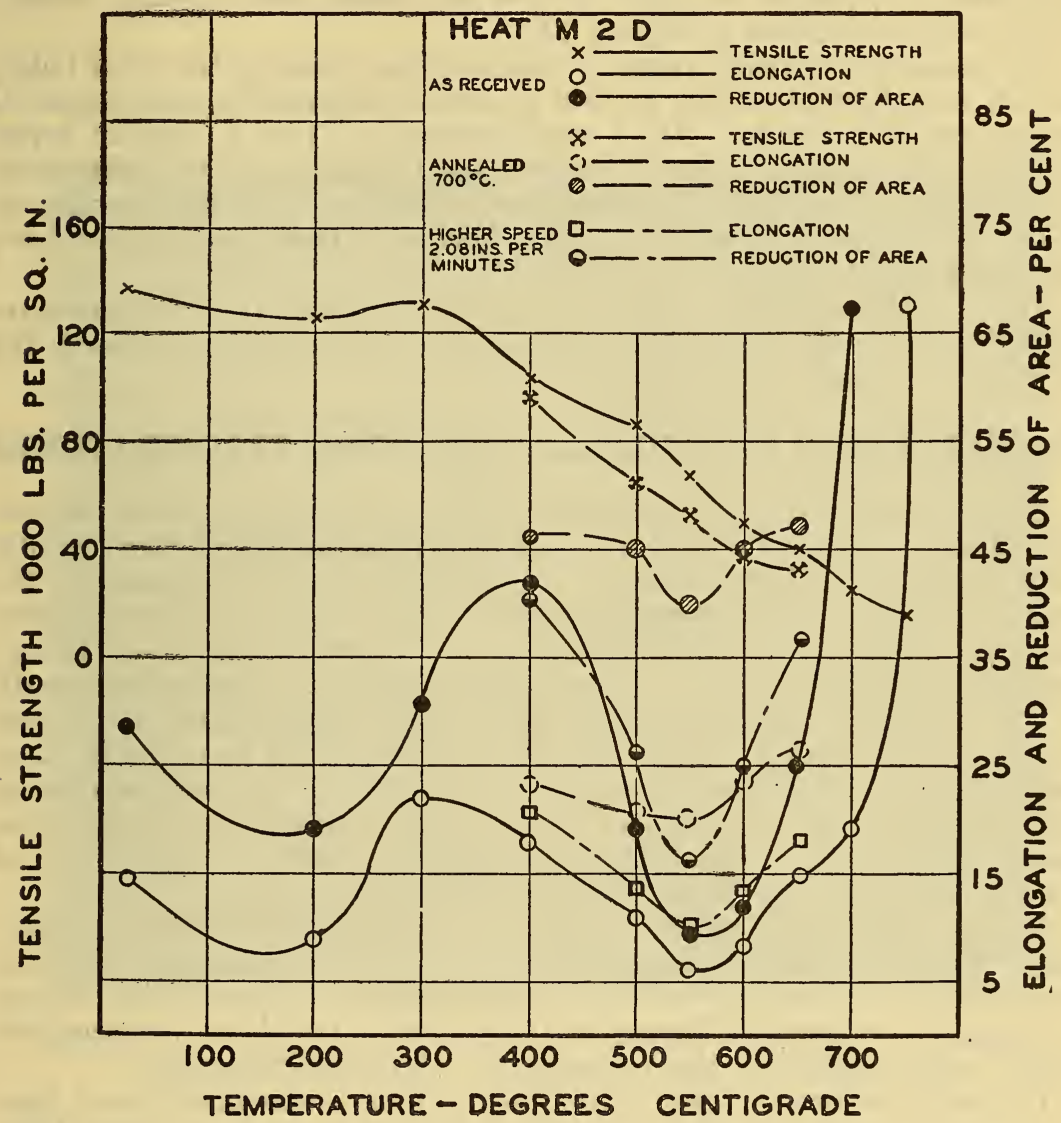

FIGURE 22.-Effect of annealing at $700^{\circ} \mathrm{C}$. and of rate of application of stress $n$ secondary brittleness in a medium manganese rail steel

similar series of tests from the rail "as rolled." It is evident that the annealing has markedly increased the ductility in the secondary brittle range, particularly the reduction of area.

A series of specimens from the $\mathrm{O}$ position of the $\mathrm{B}$ rail from heat No. 3 (fig. 3) was annealed by heating at $800^{\circ} \mathrm{C}$. for one-half hour and cooling slowly in the furnace. The results of tests of this series are included in Figure 3 . The elongation and particularly the reduction of area have been improved as a result of the annealing.

$$
98046^{\circ}-30-8
$$


It is evident from the above two series of tests made on steel from two distinctly different heats of rail steel, one of which (No. 21) was made according to "standard" practice and the other a special "killed" heat poured in hot-top ingots, that annealing the steel above the $\mathrm{A}_{c}$ transformations tends to eliminate secondary brittleness.

The effect of annealing a rail steel at a temperature above the secondary brittle range but below the $A_{1}$ transformation was then studied. A group of specimens from the O position of rail M2D was annealed by heating at $700^{\circ} \mathrm{C}$. for two hours and cooling slowly. The results are given in Figure 22.

The rather surprising result is evident that heating the steel below the $A_{1}$ transformation has caused a marked decrease in the degree of secondary brittleness. As a confirmation of this a similar series of tests was made on specimens from the $\mathrm{O}$ position of the transverse fissured rail PO (fig. 11), which had been annealed by heating to $700^{\circ} \mathrm{C}$. for two hours and cooling slowly. The results have been included in Figure 11.

It is evident that in this case also annealing at a temperature slightly below the $\mathrm{A}_{c}$ transformation has appreciably decreased the degree of secondary brittleness.

\section{NATURE OF FRACTURE AT ELEVATED TEMPERATURES}

A photograph of a series of test bars after test at the temperatures indicated is shown in Figure 4. The brittle nature of the fracture in the secondary brittle range is quite evident. The nature of the fracture at the several temperatures of test, whether intercrystalline or transcrystalline, was studied by microscopic means. Longitudinal sections were cut through the fractures parallel to the length of the specimens, polished and etched in the usual manner. It was not possible to study satisfactorily the face of the fracture in most cases because of oxidation of the fractured face during the cooling down from the temperature of test. Certain characteristic features, however, were observed in the structure just back of the fracture of specimens broken in the secondary brittle range.

In the preliminary series of tests from heats Nos. 3 and 11, a distinct "intercrystalline shattering" of the metal appeared to have occurred, as illustrated by the micrographs in Figure 23, failure occurring to a marked degree in the ferrite network or between the ferrite and pearlite at the boundary of the pearlite grains.

Figure 24 shows a longitudinal section of a specimen from heat No. 3, after testing at $650^{\circ} \mathrm{C}$., deep etched in hot concentrated $\mathrm{HCl}$. The general "shattered" appearance of the steel just back from the fracture is evident. A few cracks were present near the fracture of specimens tested at all temperatures, but they were decidedly more numerous and extended further back from the fracture in those specimens broken in their respective ranges of secondary brittleness.

The same characteristic results were obtained in similar studies made of all the series of test specimens. It may be stated that fracture in the secondary brittle range is, in general, characterized by a marked intercrystalline "shattering" not observed in tests at other temperatures. In heats in which secondary brittleness is not marked, such as heat No. 21 (fig. 7), intercrystalline failure was noted but to a much less marked extent. 


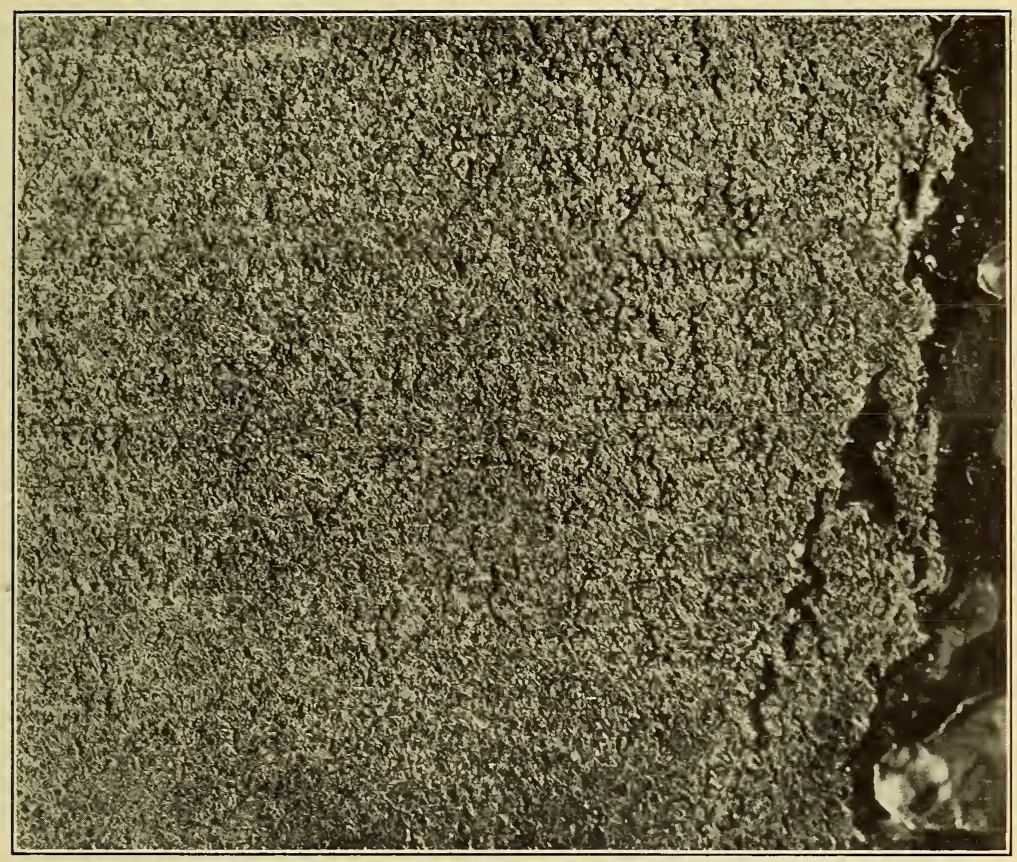

FIgURE 24.-Longitudinal section at fracture of tensile specimen from heat 3 , tested at $650^{\circ} \mathrm{C}$., deeply etched in hot concentrated $\mathrm{HCl} . \quad \times 6$ 
B. S. Journal of Research, RP164

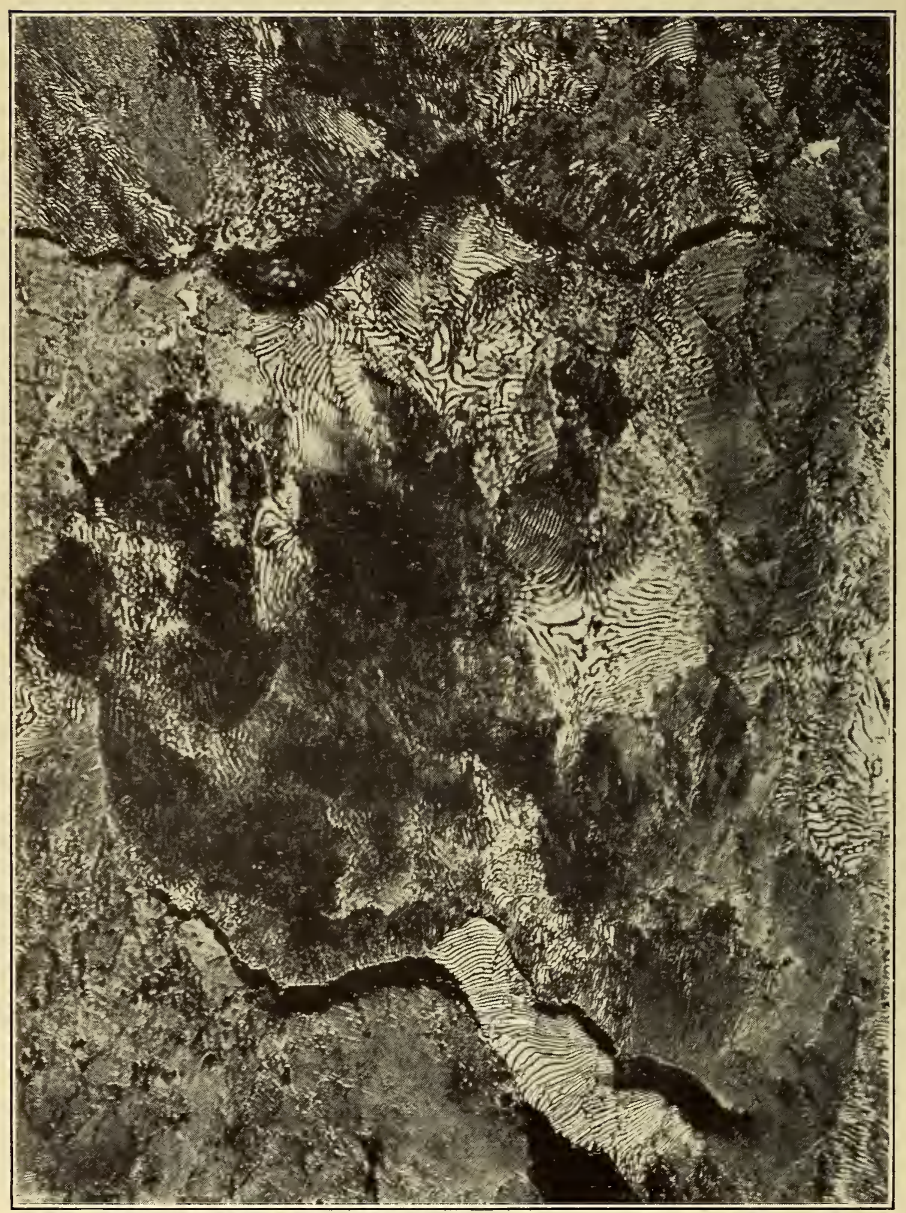

FIgURE 25.-Intercrystalline failure in tensile specimen from transverse fissured rail $\mathrm{PO}$ tested at $550^{\circ} \mathrm{C} . \quad \times 500$ 
B. S. Journal of Research, RP164

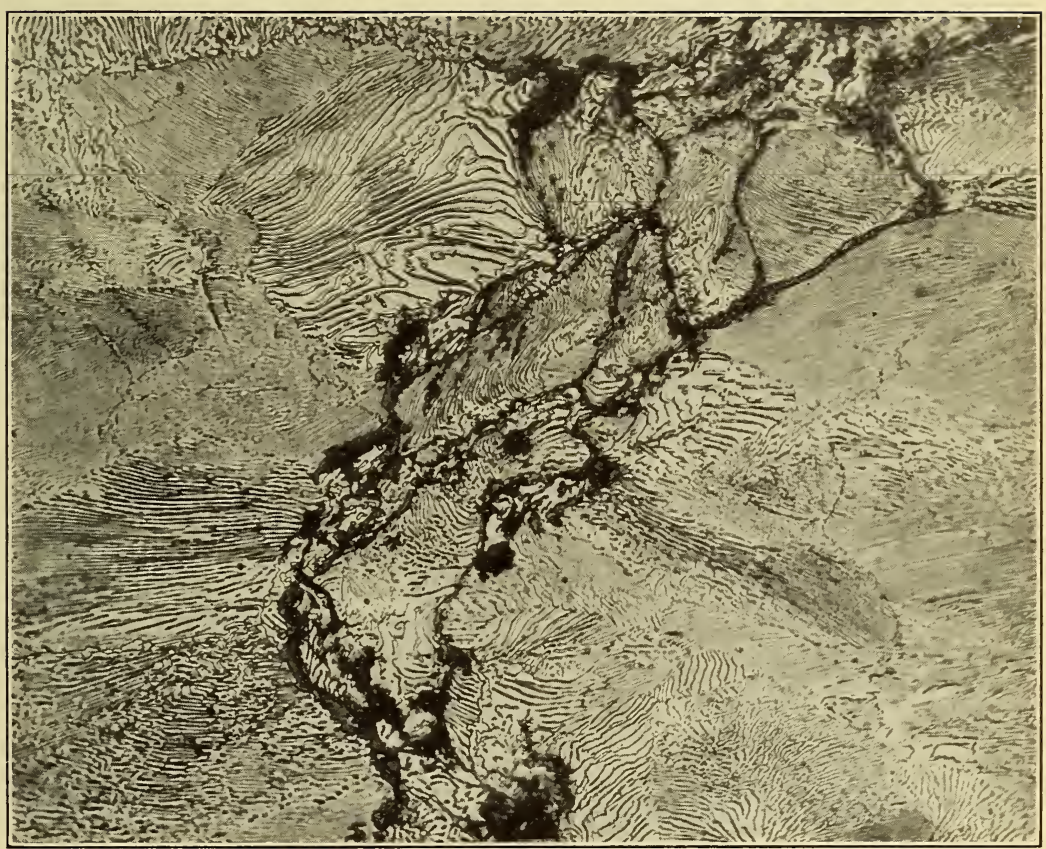

Figure 26.-Intercrystalline failure in 0.98 per cent carbon steel tensile specimen (heat $12 \mathrm{~A}$ ), tested at $500^{\circ} \mathrm{C} . \quad \times 500$ 
B. S. Journal of Research, RP164

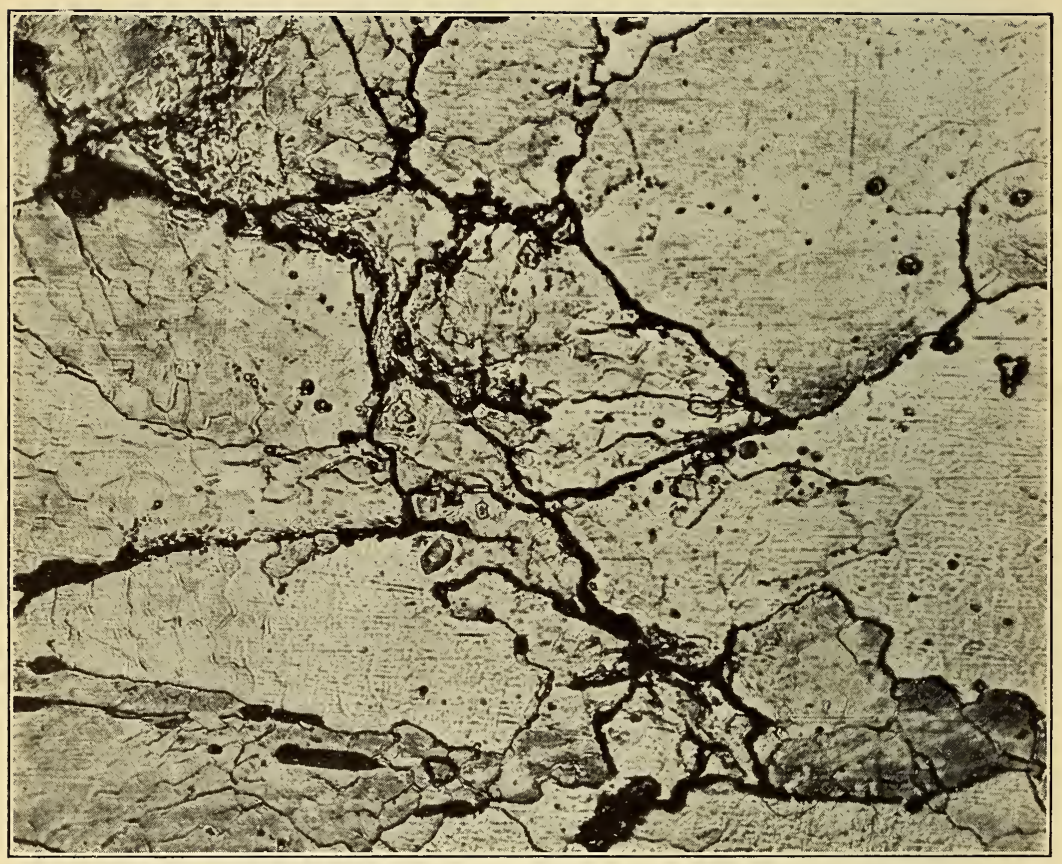

Figure 27.-Intercrystalline failure in tensile specimen of Armco iron tested at $750^{\circ} \mathrm{C} . \times 500$ 
Characteristic micrographs illustrative of the failure in the secondary brittle range of several of the steels tested are given in Figures 25 to 27. Figure 25 shows intercrystalline failure in the secondary brittle range of a specimen from a transverse fissured rail (heat PO, fig. 11). In this and other fissured rails ${ }^{17}$ intercrystalline failure had been noted in a specimen tested at normal temperatures. There is therefore some question whether the intercrystalline cracks shown in Figure 25 formed during test or previously during the cooling of the rail. There is no doubt, however, that such failure must have occurred while the metal was hot and it is a fact that intercrystalline failure was more marked in specimens from this rail broken in the secondary brittle range than in those tested at lower temperatures.

Figures 26 and 27 show characteristic intercrystalline cracks in specimens of a 0.98 per cent carbon steel and Armco iron, respectively, tested in their secondary brittle range.

The fact that intercrystalline failure is observed in the secondary brittle range of such widely diverse materials as Armco iron, rail steels and a 1 per cent carbon steel as well as some alloy steels indicates very definitely that secondary brittleness is not related to composition, but must be related to some grain boundary phenomenon common to all of the steels.

\section{TEMPERATURE DISTRIBUTION IN A SECTION OF 130- POUND RAIL UNDER DIFFERENT COOLING CONDITIONS ${ }^{18}$}

\section{TEST METHODS USED}

A rail is an unsymmetrical section. The ratio of the surface area of the base and the web to their respective volumes is appreciably greater than the ratio of surface area of the head to its volume. It follows that under similar cooling conditions the base and web of a rail would cool appreciably faster than the head. Relatively little is known regarding the magnitude of the temperature gradients that exist in a rail during cooling. Burgess and associates ${ }^{19}$ reported the results of a few determinations made on the center and inner surface of the head of a 100-pound rail cooling in air. The center of the head was found to remain about $40^{\circ}$ to $30^{\circ} \mathrm{C}$. hotter than the inner surface until the critical range was approached when the rail took on a more nearly uniform temperature throughout.

A knowledge of these gradients is of importance. They become increasingly important in view of the experiments now being carried on by some of the rail manufacturers and railroads, both in the United States and in Europe, on quenched and tempered rails.

In some recent work carried out at the Bureau of Standards, ${ }^{20}$ studies have been made of the surface and center cooling velocities of steel spheres and cylinders. Special apparatus was developed for these studies and is described in the first publication cited in the

17 Freeman, John R., jr., and Solakian, H. N., Effect of Service on Endurance Properties of Rail Steel. B. S. Jour. Research; August, 1929.

is The authors are indebted to T. E. Hamill, Bureau of Standards, for his valuable cooperation in these studies.

${ }_{10}$ Burgess, G. K., Crowe, J. J., Rawdon, H. S., and Waltenberg, R. G., Observations on Finishing Temperatures and Properties of Rails, B. S. Tech.' Paper No. 38.

${ }_{20}$ French, H. J., and Klopsch, O.Z., Quenching Diagrams for Carbon Steels in Relation to Some Quenching Media for Heat Treatment, Trans. Am. Soc. Steel Treat., 6, p. 251; 1924. French, H. J., Cook, G. S. and Hamill, T. E., Surface Cooling of Steels in Quenching, Trans. Am. Soc. Steel Treat., 15, p. 217; February, 1929 . 
reference. The principal feature of this equipment is the use of an Einthoven string galvanometer for following the extremely rapid temperature changes of a steel surface during quenching. It is capable of recording rates of temperature change of the order of several thousand degrees a second. This same equipment has been used for determining the temperature gradients that exist in a rail
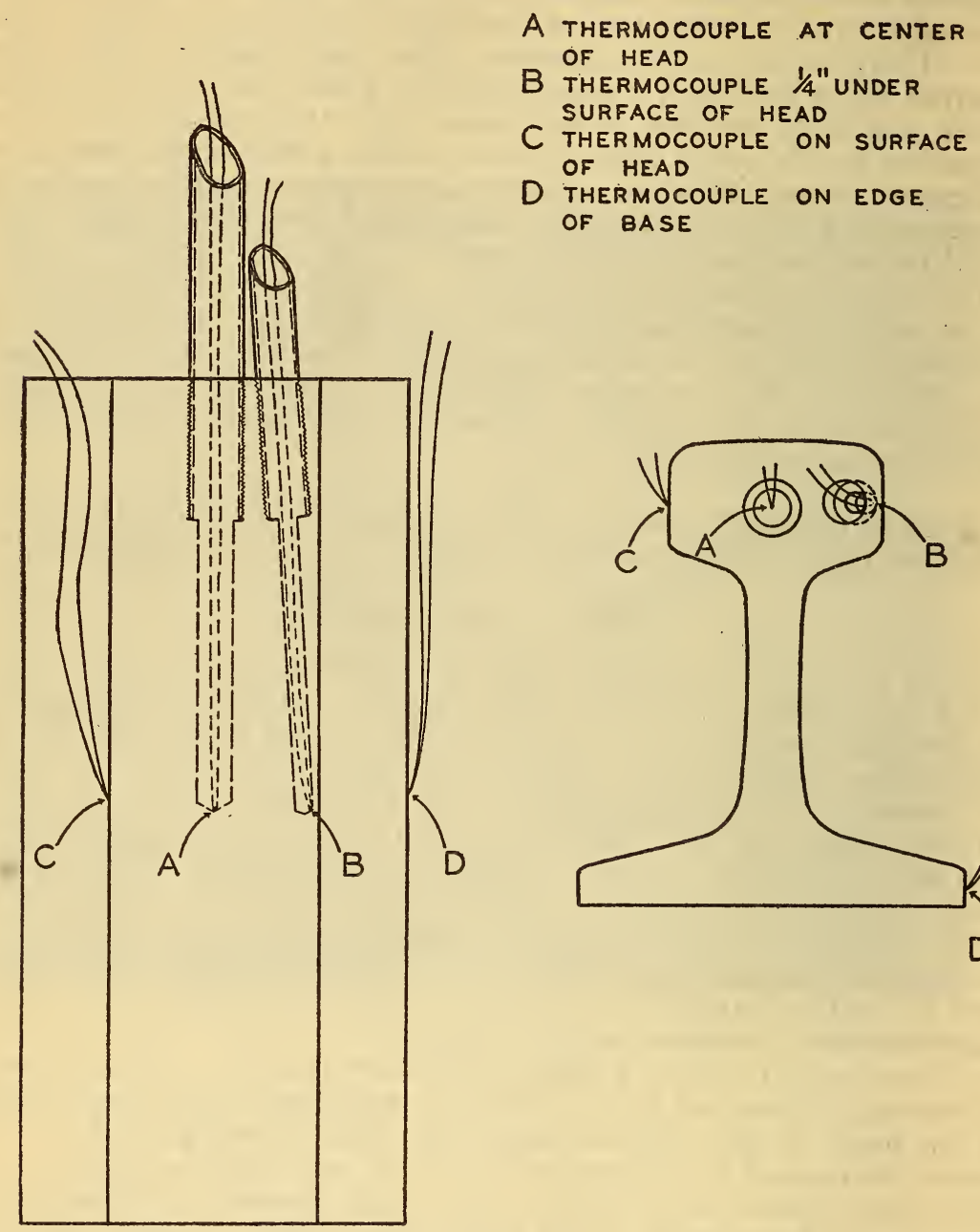

FIGURE 28.-Location of thermocouples in rail section for determining temperature gradient during cooling

under the conditions of air cooling and quenching that are reported here.

The arrangement of the thermocouples on the rail section being investigated is shown in Figure 28. A photograph of the section with thermocouples and accessory equipment for handling is shown in Figure 29. The thermocouples were welded to the rail at the point of contact. In the work referred to on cylinders this had been found 
B. S. Journal of Research, RPI64

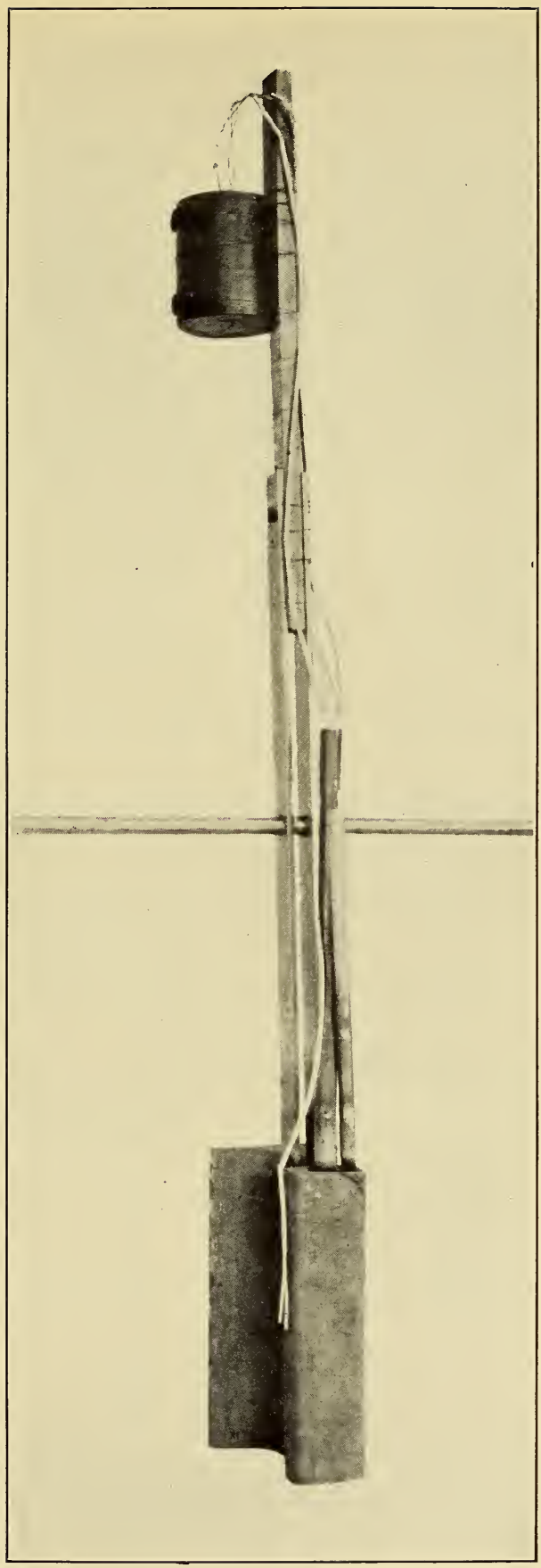

Figure 29.-Photograph of rail section showing thermocouples attached and fixtures for handling during quenching 
to be the most reliable method. A section of rail 12 inches long was used in all cases. Based on the earlier work on cylinders, it is believed that any error from end effects is negligible on a section of this length when the couples are placed at the mid section.

All tests were made on sections taken from a $\mathrm{C}$ rail of heat $\mathrm{H} 3 \mathrm{O}$. (Table 1.) In some cases, as discussed later, it was necessary to use several sections, due to breakage or cracking of the section in the quenching.

Five conditions of cooling were studied, namely, in still air, in moving air, quenching in cold water, interrupted quenching in cold water, and quenching in boiling water In all cases it was found possible to follow the center temperature during cooling with a portable potentiometer and to measure the time intervals with stop watches.

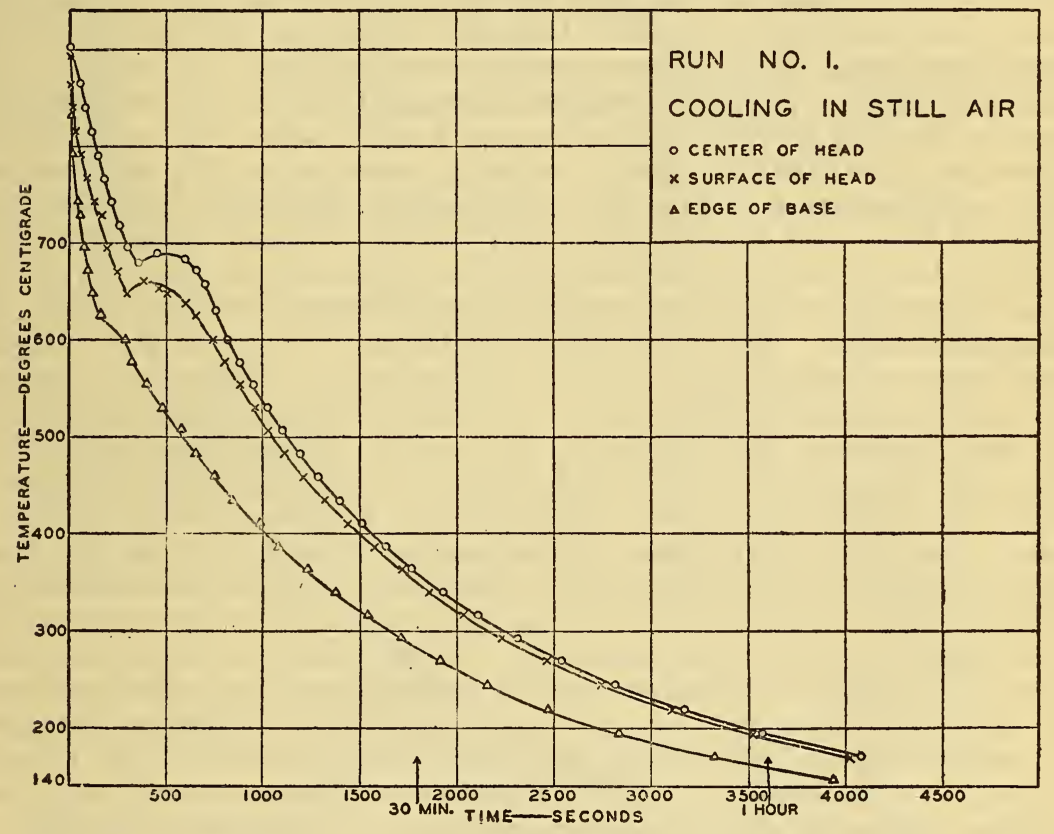

FIGURE 30.-Temperature-time curves for rail section (130-pound) cooling in still air, run No. 1

This made it possible to follow the center cooling simultaneously with the more rapid surface cooling for which the Einthoven string galvanometer was used.

\section{RESULTS OF TESTS}

\section{(a) COOLING IN STILL AIR}

The rail section with thermocouples attached was heated to about $900^{\circ} \mathrm{C}$. and held at temperature until uniformly heated throughout. The section was then removed from the furnace and suspended vertically in air about 6 inches above the floor. All windows and doors to the room were kept closed during the entire period of the cooling of the section. The temperature-time measurements were started immediately after the section was removed from the furnace. 
The temperature of the center of the head, the side of the head, and edge of the base were all recorded. The Einthoven string galvanometer was used to follow the temperature change on the edge of the base. Independent portable potentiometers and stop watches were used to follow the center and surface cooling of the head. The work of six operators and assistants was required to read and record the data. It was necessary to record the temperature-time changes at the three points independently of each other. The three temperature-time curves, however, may be plotted on the same chart. Since all three curves start from the same origin of equal temperature and zero time, a direct comparison of the temperatures existing at any of the three points after any given period of time may be made. The results are given in Figure 30.

It is evident that at the start the edge of the base and side of the head cool much more rapidly than the center of the head. With increasing time, the temperature difference between the edge of the base and the two points in the head increases until the edge of the base enters the $\mathrm{Ar}_{3}$ transformation range which causes an appreciable decrease in its rate of cooling. As soon, however, as the head enters the transformation range the temperature difference between the edge of base and both surface and center of head increases very rapidly due to the fact that the rapid rate of cooling of the edge of the base suppresses to a large extent the recalescence incident to the $A r$ transformations. The temperature difference between the surface and center of the head is not so marked as the difference between center of head and edge of the base. Following the initial rapid drop in temperature of the surface of the head to approximately $820^{\circ} \mathrm{C}$., the rates of cooling of center and surface are approximately the same until the surface enters the transformation range at about $645^{\circ} \mathrm{C}$., when, due to the temperature of the surface increasing while the center is continuing to cool, the surface temperature approaches the center temperature. As soon, however, as the center enters the transformation range its temperature increases. The surface, however, has now passed through its recalescence and is again decreasing in temperature. A resultant temporary increase in temperature difference between center and surface, therefore, occurs. After the center has passed through its recalescence, this temperature difference gradually decreases with a simultaneous decreasing rate of temperature change as the rail sect a s proaches normal temperatures.

The maximum difference in temperature between center and surface of he accers when the surface is at about $830^{\circ} \mathrm{C}$. and the center at about $895^{\circ} \mathrm{C}$, almost immediately after removal of the rail from the furnace ard probably at the instant a decrease in temperature first occur ed in the center.

The miximum siference in temperature between the edge of the base and the surfare of the head apparently occurred shortly after the latter had passed through the transformation range. At this instant, the temperature of the edge of the base was about $470^{\circ} \mathrm{C}$. and the surface of the head lout $610^{\circ} \mathrm{C}$., a temperature difference of about $140^{\circ} \mathrm{C}$. At the same instant the temperature of the center of the head wa about $660^{\circ} \mathrm{C}$., about $50^{\circ} \mathrm{C}$. higher than the surface. The center had al:o just merged from the transformation range.

The fict that a large temperature difference may exist in a rail during cooling when at the above temperatures appears of significance 
when it is noted that the secondary brittle range in rail steels has been found to occur in the same temperature range. As discussed more fully later in this report the large temperature gradients existing may set up internal tensile stress which may cause internal failure in the secondary brittle range.

\section{(b) COOLING IN MOVING AIR}

A similar series of curves was obtained on the same rail section when cooling in moving air. In this case a current of air from an electric fan having four 8-inch blades was directed on the head of the rail during cooling. The fan was 5 feet from the head of the rail. The results obtained are given in Figure 31 .

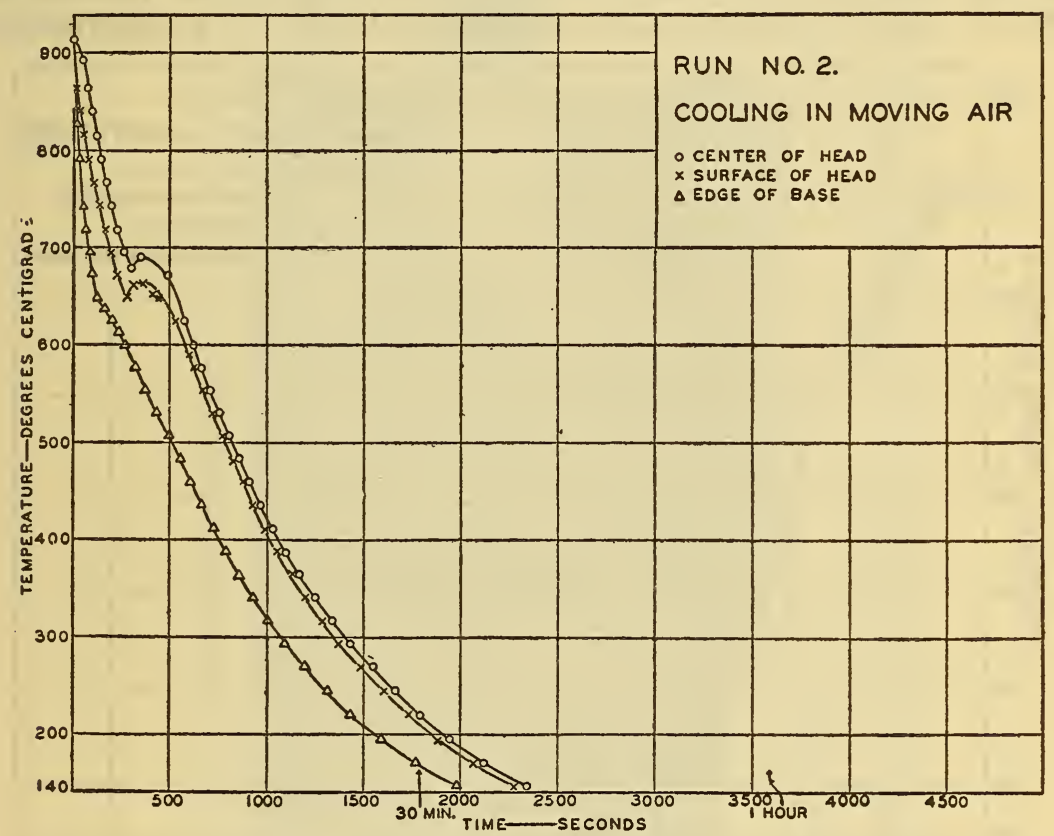

FIGURE 31.-Temperature-time curves for rail section (130-pound) cooling in moving air, run No. 2

It is evident that the general relation of the cooling velocities of the three points to each other is approximately the same as in still air. The total time of cooling, however, is considerably less in moving air. In still air the total time required for the center of the head to cool from $900^{\circ}$ to $200^{\circ} \mathrm{C}$. was 3,500 seconds while in moving air the total time required from $910^{\circ} \mathrm{C}$. was only 1,900 seconds, or only about 54 per cent as long. The relative cooling times of the surface of the head and edge of base were in proportion.

In still air the center cooled from $900^{\circ} \mathrm{C}$. to the temperature of the $A r_{3}$ transformation at $680^{\circ} \mathrm{C}$. in 350 seconds while in the moving air it cooled from the slightly higher temperature of $910^{\circ} \mathrm{C}$. to the transformation temperature in 300 seconds.

There was a marked difference in the time required to cool through the transformation range at the respective positions in the rail in 
still air as compared to the conditions of moving air. In still air the temperature of the center remained above the temperature at which recalescence started $\left(680^{\circ}\right.$ C.) for 250 seconds while in moving air the corresponding time was only 160 seconds. The temperature rise, however, during recalescence was about the same $\left(10^{\circ}\right.$ C.) in each instance.

The maximum temperature difference between surface and center of the head when cooling in moving air was practically the same $\left(65^{\circ}\right.$ C.) as in still air and also occurred shortly after cooling first started in the center. Also the difference decreased but slightly until the surface entered the transformation range.

Similarly the maximum difference in temperature between edge of base and surface of head occurred while cooling in moving air shortly after the surface of the head emerged from the transformation

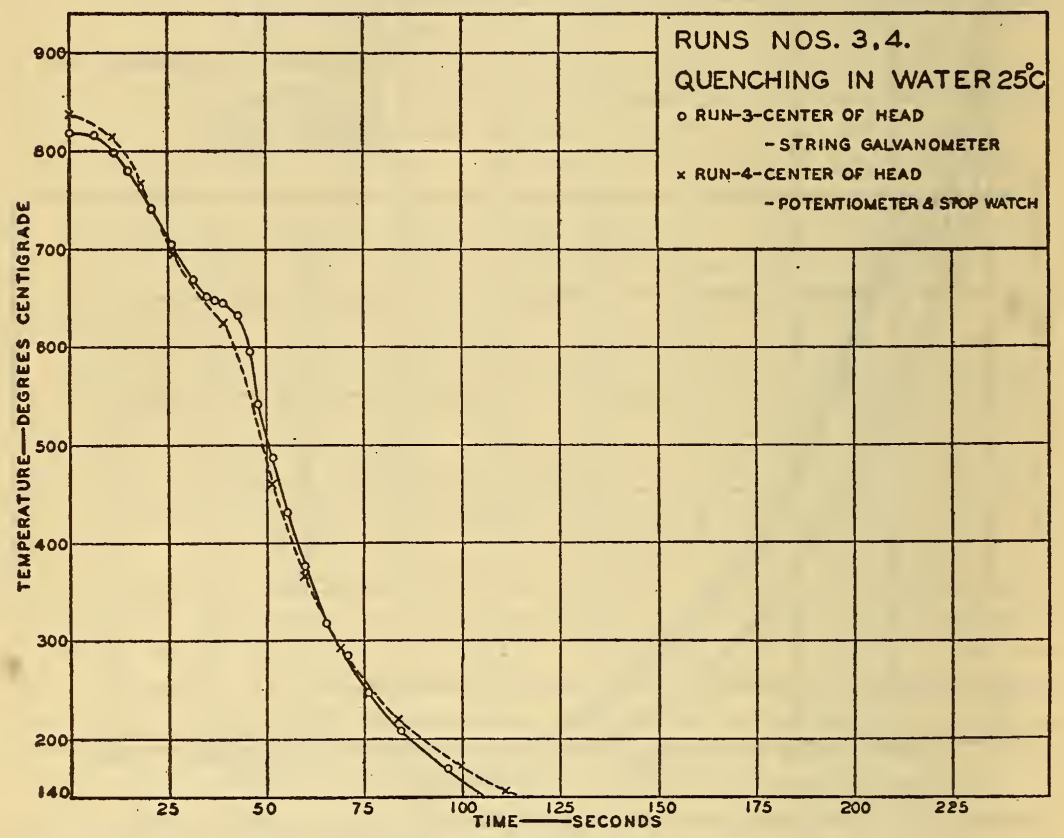

FIgURE 32.-Temperature-time curves of center of rail section (130-pound) quenched in water, runs Nos. 3 and 4

range which was found under the condition of cooling in still air. The temperature difference was practically the same in each instance, being $140^{\circ} \mathrm{C}$. for still air and $135^{\circ} \mathrm{C}$. for moving air.

It is evident that the principal effect of the air stream from the fan blowing over the head of the rail section was to cause a more rapid cooling. The temperature distribution in the section, however, was practically the same. Any stress developed as a result of temperature gradients would, therefore, probably be about the same for both conditions of cooling.

(c) QUENCHING IN COLD WATER

The same section was used for this test as was used in the previous two series. It was thought that the center cooling velocity of the head would be too great to follow with the potentiometer and stop 
B. S. Journal of Research, RP164

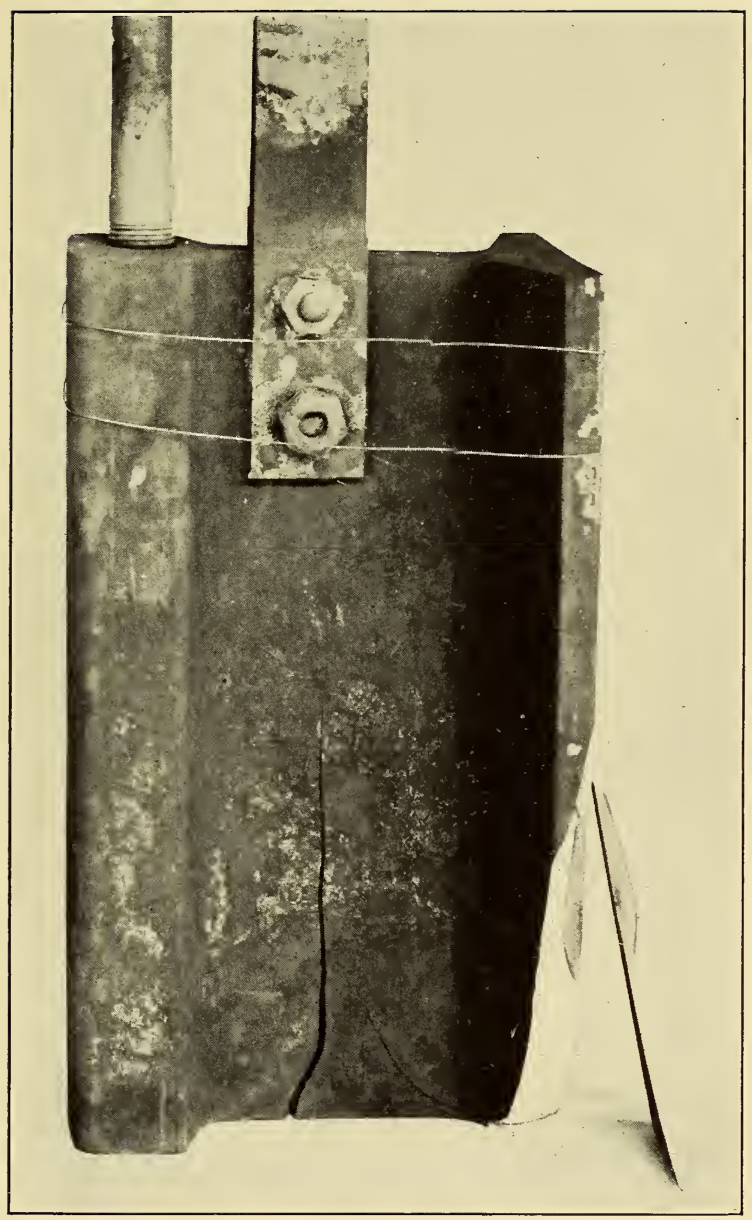

FIGURE 33.-Rail section after quenching in water showing fracture 
watches. Therefore, in the first run of this series (No. 3) the center cooling velocity was determined using the Einthoven string galvanometer. The section was heated to a uniform temperature of about $820^{\circ}$ C. It was then quenched in tap water at $25^{\circ}$ C. During quenching the section was moved up and down in the water.

The temperature-time curve is given in Figure 32 (run No. 3).

A similar quench was made on this same section in which the center cooling was followed by a portable potentiometer and stop watches. The results have been included in Figure 32 (run No. 4) for comparison with the previous run. The initial temperature of the section, $840^{\circ} \mathrm{C}$., previous to quenching was about $20^{\circ} \mathrm{C}$. higher than in the previous run. The temperature of the water before

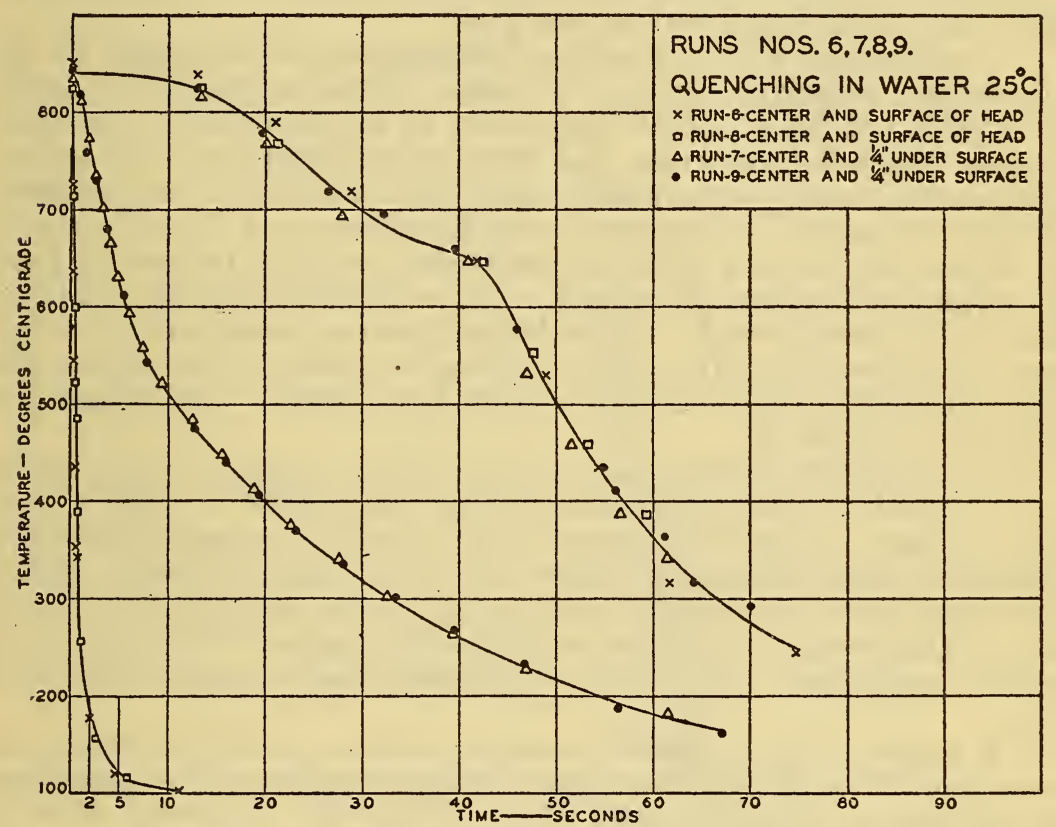

FIGURE 34.-Temperature-time curves of surface, center, and intermediate position in head of rail during quenching in cold water, runs Nos. 6, 7, 8, and 9

quenching was $28.5^{\circ} \mathrm{C}$. and after quenching had risen in the upper portion of the quenching tank to about $43^{\circ} \mathrm{C}$.

The agreement of the two cooling curves is very good. The greater sensitivity of the autographic string galvanometer in indicating the temperature changes is evident, particularly in showing the heat effect while passing through the transformation range.

The rail section was removed from the water when the center of the head was slightly less than $100^{\circ} \mathrm{C}$. A large crack had formed, during the quenching, at the lower end of the web and a small one was apparent on the edge of the base. While examining the rail several minutes after removing from the quenching water a piece of the base broke off with appreciable force indicating the presence in the rail after quenching of residual internal stresses of appreciable magnitude. Figure 33 shows the crack in the web and the fracture and piece that broke off of the base. The dark area in the fracture 
indicates the depth of crack in the base that probably formed during quenching and acted as the nucleus for the failure that occurred after quenching. A temperature-time curve of the surface of the head was not obtained in this run due to breaking of the couple.

For further tests a similar section was prepared from an adjacent section of the same rail. Temperature-time curves for the center and surface of the head of this section during quenching are given in Figure 34 (run No. 6). The section was quenched at $850^{\circ} \mathrm{C}$. into water at $25^{\circ} \mathrm{C}$. The extremely rapid cooling of the surface is apparent. The total time of cooling from the quenching temperature of $850^{\circ}$ to $300^{\circ} \mathrm{C}$. was 0.6 second as compared to 66 seconds required for the center to cool to the same temperature.

A duplicate run, No. 8, was made, and results are included in Figure 34. The agreement is very good.

It was thought that the surface temperature of the head of the rail section, especially during the rapid cooling incident to quenching, might not be a reliable indication of the temperature a short distance beneath the surface and that the temperature in the underlying metal would more nearly approach the center temperature. Cooling curves were, therefore, taken simultaneously during quenching of this section, at a point approximately one-quarter inch in from the surface of the head of the rail. The method of locating the junction of the thermocouple in the desired position was similar to that used for determining the center cooling as indicated in Figure 28. Two independent runs (Nos. 7 and 9) were made. The results are included in Figure 34.

The curves were all obtained on the same rail section. The quenching temperature was approximately the same $\left(830^{\circ}\right.$ to $840^{\circ}$ C. $)$ in each instance. The temperature of the quenching water at instant of quenching was between $25^{\circ}$ and $28^{\circ} \mathrm{C}$. in each instance. After quenching it was between $32^{\circ}$ and $35^{\circ} \mathrm{C}$. in the upper portion of the tank. The center cooling was followed in each of the four independent quenches. The curve is therefore the average of the four runs.

It is evident from the curves that the surface cooling is very much more rapid throughout the entire cooling range from the quenching temperature of approximately $835^{\circ}$ to $250^{\circ} \mathrm{C}$., the total time of cooling being about 0.8 second as compared to about 75 seconds required for the center and 43 seconds for the intermediate position.

The cooling rate at the intermediate position more nearly approximates the surface than the center cooling rate for temperatures above the transformation. The temperature at which the Ar transformations occur in the intermediate position is not well defined. A change in the rate of cooling becomes manifest however, at approximately $620^{\circ} \mathrm{C}$. With further decrease in temperature the cooling rate decreases rapidly. The center cooling rate is relatively very slow during the first few seconds of cooling. Eleven seconds after quenching the surface temperature of the head has dropped to $100^{\circ} \mathrm{C}$. while the center has decreased less than $10^{\circ} \mathrm{C}$., giving an instantaneous temperature difference of over $730^{\circ} \mathrm{C}$. At the same instant the intermediate position has a temperature of $490^{\circ}$, or more than $340^{\circ} \mathrm{C}$. below the center temperature. The occurrence of the $\mathrm{Ar}_{3}$ transformation is relatively well defined in the center cooling curve beginning at approximately $680^{\circ} \mathrm{C}$. The rate of cooling 
decreases appreciably during the transformation. Immediately following the transformation the center cooling is very rapid relative to the intermediate position.

The approximate temperature distribution that exists in the head of the rail after certain definite intervals of time during quenching is given in Figure 35. This plot is obtained by scaling from the curves given in Figure 34 the temperature values at the selected time intervals and plotting them as ordinates against distance from center of railhead toward surface as abscissæ. Three points only are available to indicate temperature distribution at any given time interval. These points have been connected by straight lines, although the temperature probably changes at a gradually increas-

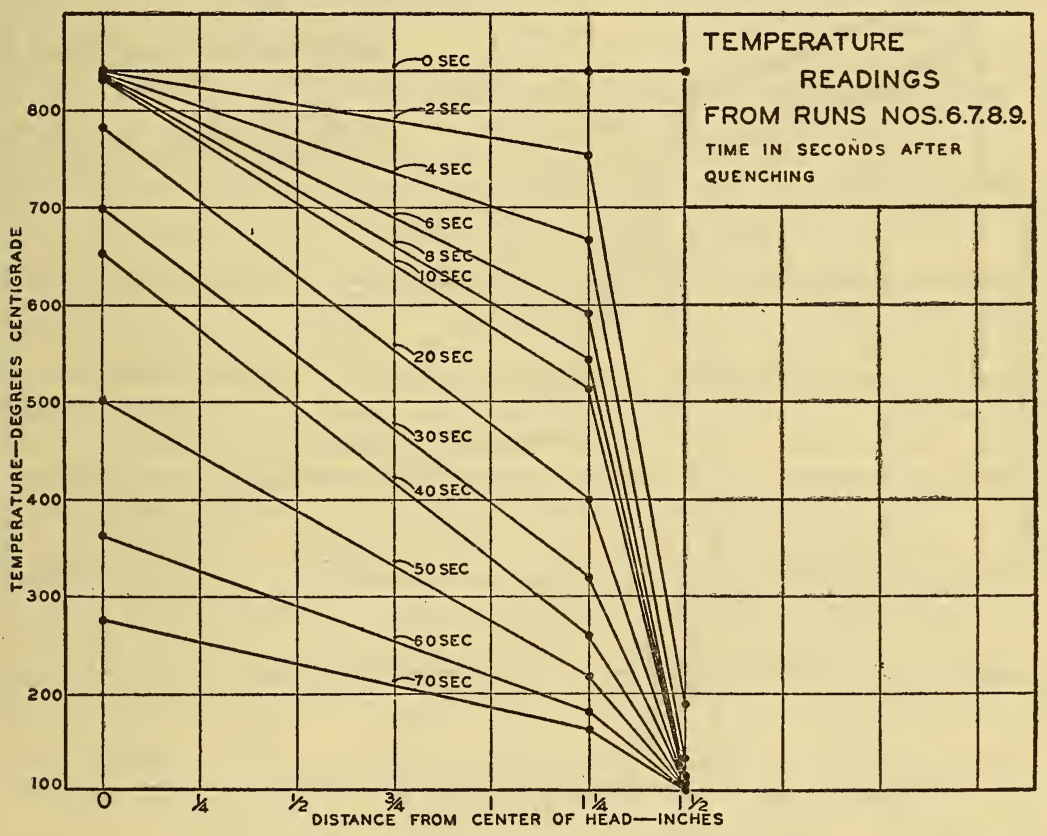

FIGURE 35.-Temperature distribution in head of rail section (130-pound) during quenching in cold water

ing rate toward the surface. The plot indicates, however, the very sharp temperature gradient that exists, especially during the first few seconds of cooling, between the surface of the head and the underlying position only one-quarter inch below the surface as compared to the gradient existing between this underlying position and the center. It also indicates, as cooling progresses, the rapid increase in temperature gradient between the position under the surface and center. The magnitude of this gradient throughout the greater part of the cooling period is also apparent.

The cooling rate of the base during quenching was not determined. In view of the fact that in air the edge of the base cooled more rapidly than the surface of the head it would also undoubtedly cool more rapidly during quenching. 
(d) INTERRUPTED QUENCHING

Kenney ${ }^{21}$ has described a special quenching procedure said to be especially applicable to rails. This process consists essentially in hardening the steel rail by quenching the rail in water to a temperature below the critical range and then before it has cooled to a temperature as low as the blue-heat zone to thoroughly equalize the temperature throughout the section after which it may be cooled to atmospheric temperature. Kenney states that internal and external rupture occurs if the rail is allowed to cool to the blue-heat range without equalizing the temperature. That external rupture may occur is confirmed by the results obtained in the present work

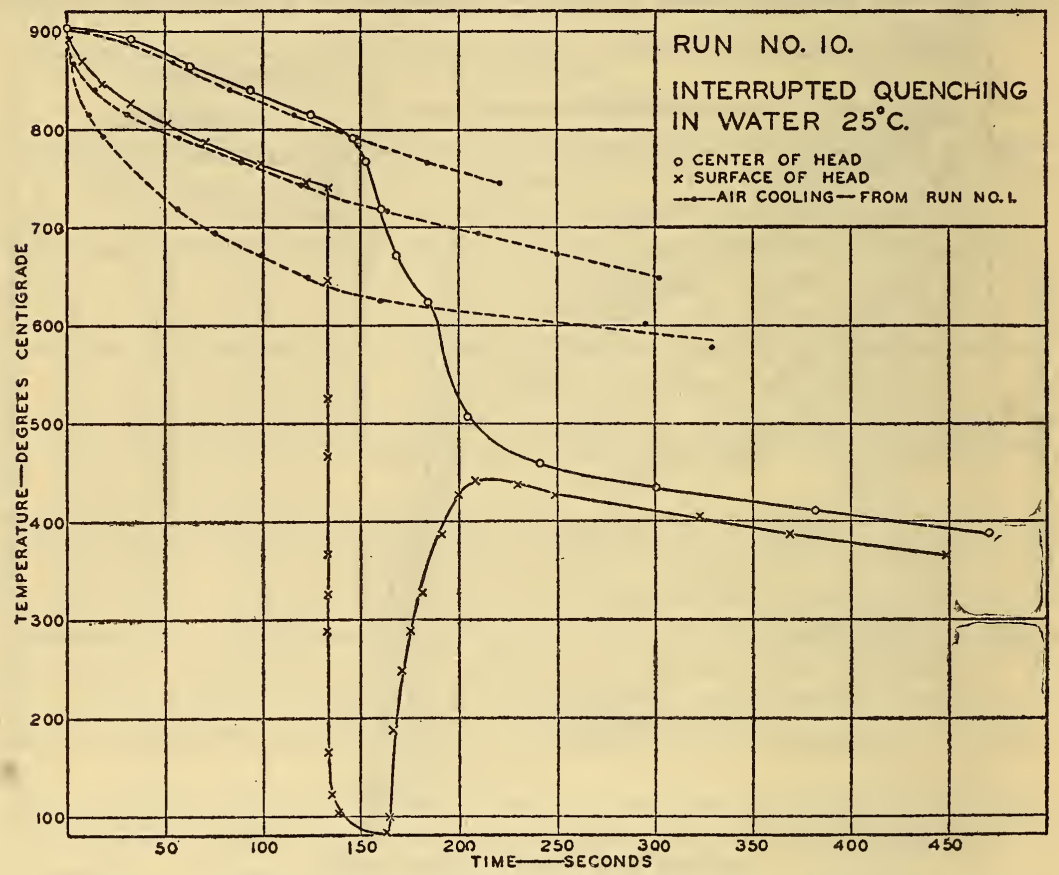

FIgURE 36.-Temperature-time curves for rail section (130-pound) during interrupted quenching in water, run No. 10

(run No. 4) where it was found that the web and base of a short section of rail may break (fig. 33) when quenched in water. It is also stated by Kenney that even if a rail is quenched for only 30 seconds and then allowed to cool in air, internal as well as external rupture will occur.

It was believed of interest to determine the temperature gradients in the head of a rail during an interrupted quench. Accordingly, a new section of rail was taken from an adjacent position in the same rail from which the previous two sections were taken. The dimensions of this section and arrangement of thermocouples were the same as in the previous runs.

The section was heated to $905^{\circ} \mathrm{C}$. It was then removed from the furnace and held in air above the quenching bath until the edge of

${ }^{21}$ Kenney, E. F., Heat Treatment of Steel, U. S. Patent No. 1619025; Heat Treatment of Railway Rails, Reissue 17240. 
the base showed magnetism as determined with a small strong hand magnet. The section was then quenched in water for 30 seconds. It was then withdrawn from the quenching bath and allowed to cool in air to normal temperatures. Temperature-time curves of the center and surface of the head were determined from the time the section was removed from the furnace until several minutes after the section was removed from the quenching bath. The results of this run, No. 10, are given in Figure 36.

The three distinct phases of cooling are apparent: Cooling in air until edge of base became slightly magnetic, cooling during quenching in water, and cooling in air after quenching. For ease of comparison a portion of the temperature-time curves obtained on the similar section during cooling in still air (run No. 1, fig. 30) have been reproduced on the larger scale of Figure 36. As would be expected, the center and surface cooling curves of the two sections are practically the same during the initial period of air cooling. Judging from the temperature-time curve of the edge of the base obtained in the previous run, appreciable magnetism appeared when the temperature of the edge of the base was at approximately $645^{\circ} \mathrm{C}$. (Judging from color the temperature of this surface was considerably less.) The temperatures of the center and surface of the section were, at moment of quenching, $810^{\circ}$ and $740^{\circ}$ C., respectively. The extremely rapid rate of cooling of the surface of the head during quenching is apparent, and is comparable to that obtained in the previous runs. The surface temperature cools from $740^{\circ}$ to $200^{\circ} \mathrm{C}$. in less than one second. There is no marked change in the rate until about $120^{\circ} \mathrm{C}$. is reached. During the remaining period of the quench, about 28 seconds, the surface temperature cools to a minimum of $82^{\circ} \mathrm{C}$. On removal from the quenching bath the surface temperature rises very rapidly, attaining a maximum temperature of $444^{\circ} \mathrm{C}$. in 45 seconds, and then again decreases continuously at a relatively slow rate.

During the 30 seconds that the rail section was in the quenching water the center temperature decreased from $810^{\circ} \mathrm{C}$. to about $700^{\circ} \mathrm{C}$. and, it is of interest to note, had apparently not entered the $A r_{3}$ transformation range. During the period that the surface temperature was rapidly increasing after removal from the quenching water the center temperature continued to decrease rapidly through the $A r_{3}$ transformation range, the central portion of the head giving up its heat to the cooler exterior. At about $470^{\circ} \mathrm{C}$. a condition of equilibrium appears to be established and center and surface cool at about the same rate with a temperature difference of approximately $25^{\circ} \mathrm{C}$.

Equipment was not readily available for equalizing the temperature of the section after quenching by transferring back into a furnace at approximately $510^{\circ} \mathrm{C}$. $\left(950^{\circ} \mathrm{F}\right.$.), as recommended by Kenney, and thereby having the temperature equalized throughout the section. Had this been done it is obvious from the results given in Figure 36 that the surface temperature would have increased after quenching at a somewhat more rapid rate. It is difficult to estimate the effect on the center cooling. It would seem to depend largely upon the time required to transfer the section from the quenching bath into the furnace. If this were done instantaneously the rate of cooling of the center through the transformation range might, due to a more rapid rise in surface temperature, be appreciably decreased. If 15 seconds were required to complete the transfer, the center, as 
may be seen from the curves would already have passed through the transformation. Had the section been quenched for a shorter period of time, for example, 15 seconds, the rate of cooling of the center through the transformation range would apparently have been appreciably slower, even with air cooling of the section after quenching, and if the section were reheated after quenching the rate would conceivably have been so slow as not to produce appreciable hardening. This indicates quite definitely that a rather critical relation exists between time in quenching bath, time required to transfer from quenching bath to a reheating (equalizing) furnace, and resultant hardness of the center of the head of the rail.

\section{(e) QUENCHING. IN BOILING WATER}

A somewhat less drastic quench than in cold water would be desirable for rails in order to eliminate the excessive temperature gradients and the accompanying stresses. Oil might be suitable as a quenching medium, but because of the large volume required and difficulties of handling as well as cost would probably not be feasible for treating a tonnage production such as rails.

It is a well-known fact that the rate of cooling of a steel object is considerably slower in hot water than in cold water. In a recent report ${ }^{22}$ it has been shown that water at $60^{\circ} \mathrm{C}$. gives a cooling rate intermediate between oil and water and might be suitable, but the difficulties of maintaining at a prescribed temperature the large volume of water that would be required in the production of rails would be difficult. In the same report boiling water is not considered so desirable a quenching medium as some others. It does give, however, an intermediate cooling rate between still air at $20^{\circ} \mathrm{C}$. and oil at $20^{\circ} \mathrm{C}$. and it would be relatively easy to maintain its temperature at or very near the boiling point in the mill.

It was, therefore, believed of sufficient interest to determine the center and surface cooling speeds of a rail section when quenched in boiling water. Two runs (Nos. 11 and 12) were made in which a section similar to that used in the intermediate quenching experiments was quenched in boiling water. The results are given in Figure 37. The temperature of quenching was about $835^{\circ} \mathrm{C}$.

It is evident from the curves that the cooling rate, especially of the surface, is very much less than in water at $20^{\circ} \mathrm{C}$., but appreciably faster than in air. The $A_{r}$ transformations are evident in the surface temperature-time curve as well as in the center one. The rate of cooling of the latter, however, was relatively so slow that it appeared questionable whether any worthwhile increase in hardness would be obtained as compared to present practice. A section was, therefore, cut from the middle portion of the quenched section, and the hardness found to be as given in the following table, in which hardness previous to quenching is also given for comparison:

\begin{tabular}{|c|c|c|c|c|}
\hline & \multicolumn{4}{|c|}{ Hardness } \\
\hline & \multicolumn{2}{|c|}{$\begin{array}{l}\text { Rockwell B posi- } \\
\text { tion }\end{array}$} & \multicolumn{2}{|c|}{$\begin{array}{l}\text { Brinell } 3,000 \mathrm{~kg} \\
\text { load position }\end{array}$} \\
\hline & 0 & $\mathrm{M}$ & 0 & M \\
\hline $\begin{array}{l}\text { Rail H3C as rolled } \\
\text { Rail H3C quenched in boiling water }\end{array}$ & $\begin{array}{l}103 \\
107\end{array}$ & $\begin{array}{l}103 \\
107\end{array}$ & $\begin{array}{l}285 \\
341\end{array}$ & $\begin{array}{l}285 \\
321\end{array}$ \\
\hline
\end{tabular}

${ }_{22}$ French, H. J., and Hamill, T. E., Hot Aqueous Solutions for the Quenching of Steels, Preprint No. 8, A. S. S. T.; 1929. 
Apparently some increase in hardness was obtained by reheating and quenching in boiling water. Further tests must be made, however, before definite conclusions may be drawn. It should also be pointed out that reheating and quenching would probably give higher values than would be obtained by quenching directly after rolling.

\section{DISCUSSION OF RESULTS WITH SPECIAL REFERENCE TO SHATTER CRACKS IN RAIL}

Waring and Hoffammann ${ }^{23}$ have shown the presence of internal cracks in both new and failed rails and forgings, and suggested that

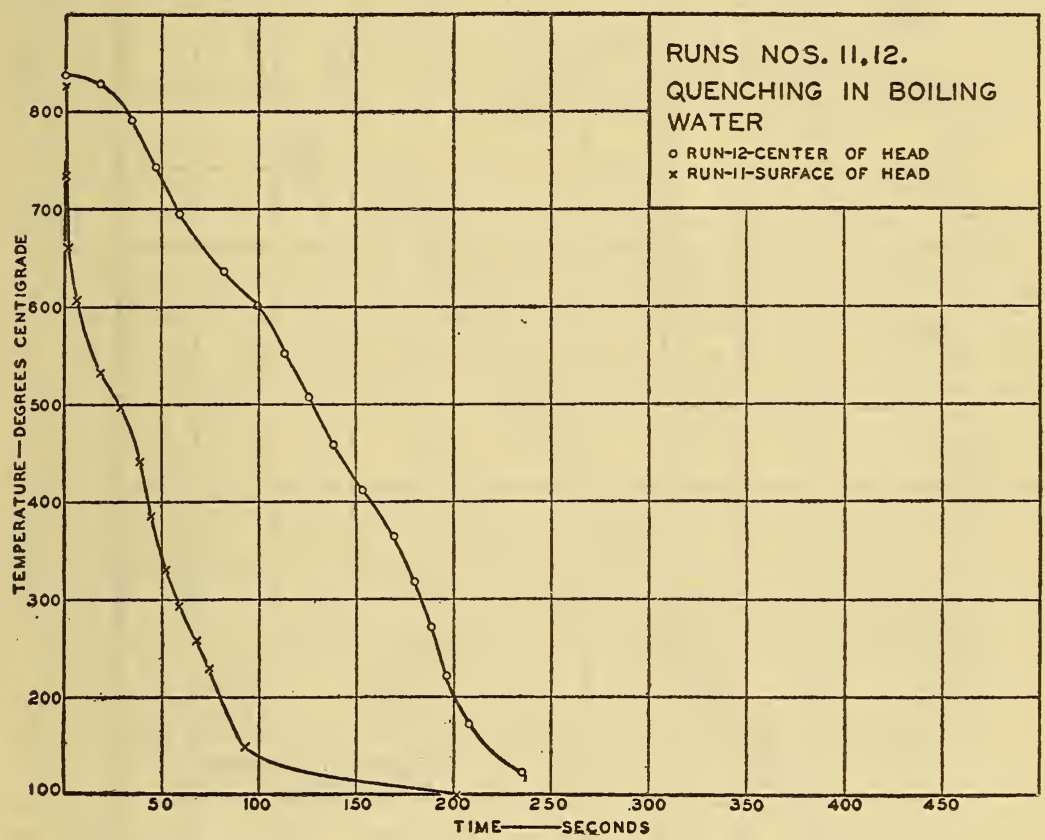

FIgURE 37.-Temperature-time curves for rail section (130-pound) during quenching in boiling water, run No. 12

these cracks might be the nucleus from which transverse fissure failures in rails develop. The cracks were found to be both intercrystalline and transcrystalline in character and were believed by the authors to develop during some stage in the process of fabrication of the material.

Howard in his discussion of their paper states that the cracks probably occur when the steel is at a fairly high temperature. He pointed out in this and in a later report ${ }^{24}$ that internal strains of tension develop in cast, rolled, or forged shapes on cooling; that this tensile stress may be along each of its three axes; that the shattering may be

${ }^{23}$ Waring, F. M., and Hoffammann, K. E., Deep Etching of Rails and Forgings, Proc. A. S. T. M., 19, Pt. II, p. 182, 1919.

${ }_{24}$ Interstate Commerce Commission, Report of the Director of the Bureau of Safety in regard to investigat1on of an accident which occurred on the St. Louis-San Francisco Ry. near Victoria, Miss., on Oct. 27, 1925. Report dated Jun 16 , 1927. 
related to the rate of cooling; and that shrinkage cracks represent the relief of the strains by reason of the rupture of the metal.

It has been stated ${ }^{25}$ that the shattered zones found in rails do not extend to the ends of the rail indicating that the time of occurrence is after the rail length is cut at the hot saw.

There is shown in Figure 38 the thermal expansion and contraction curves of a steel of rail-steel composition. It may be seen that the

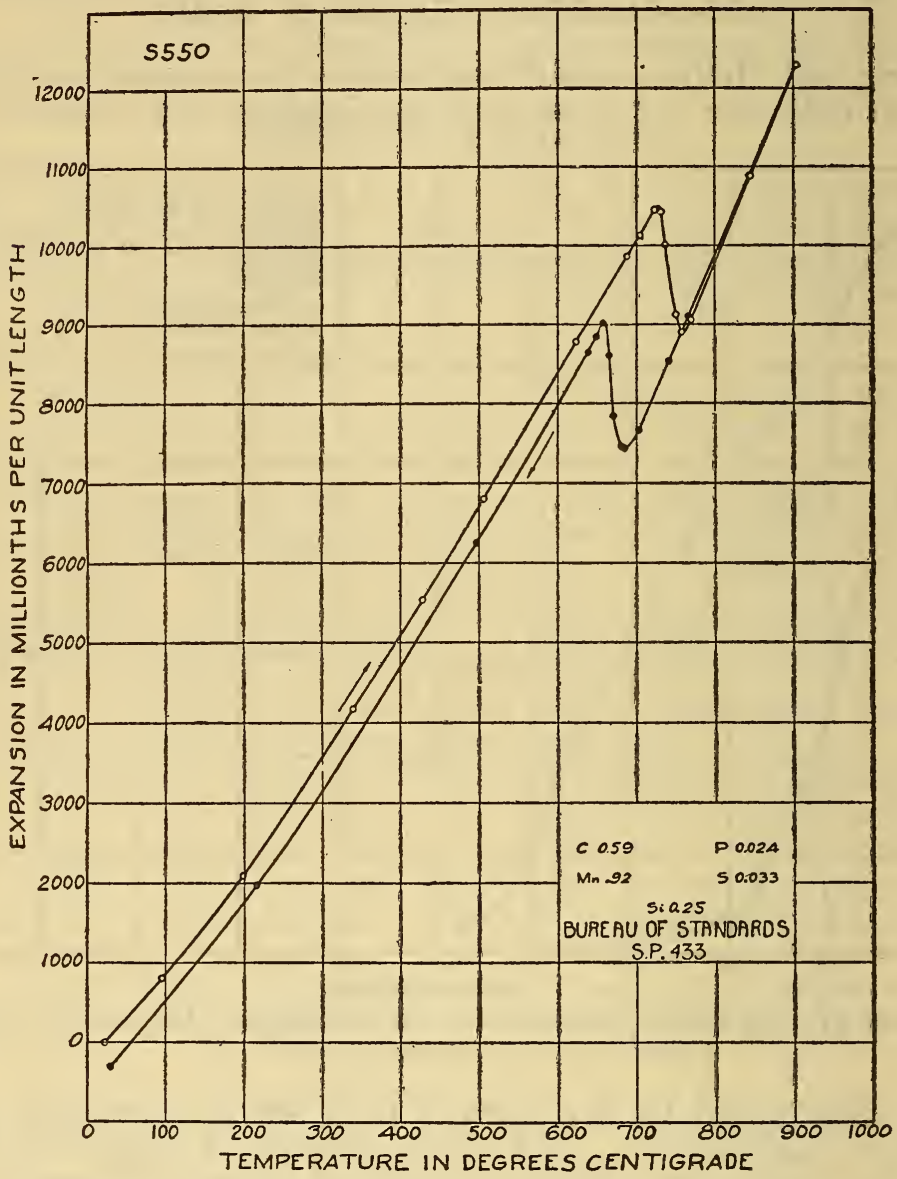

FIGURE 38.-Thermal expansion curve of steel of rail composition

steel contracts on cooling at an approximately uniform rate to $685^{\circ} \mathrm{C}$. where a marked expansion occurs. With further cooling the steel again contracts at an approximately uniform rate until normal temperatures are reached. This dilation of steel is a well-known phenomenon. It is associated with the transformation of gamma iron to alpha iron. It will occur in all rails of normal composition, the tem-

${ }^{25}$ Wickhorst, M. H., The Relation of Shattered Steel in Fissured Rails to the Mill End of the Rail, Proc. A. R. E. A., 22; p. 216, 1921. Howard J. E., On Shattered Zones in Certain Steel Rails, Proc. A. S. T. M., 20, Pt. 2, p. 44; 1920. 
perature depending principally on the carbon and manganese content and the rate of cooling.

Consider the cooling of a rail after leaving the hot saw. The rail is generally given a predetermined camber with the head convex, this having a radius of curvature which experience has shown will cause the rail to be approximately straight when cold. The rail is then placed on the hotbed and starts to cool with its head convex. By referring to Figures 30 and 31 , it is seen that as a rail section cools in air the base cools more rapidly than the head. According to Figure 38 the base will, therefore, contract more rapidly than the head. This contraction acts to increase the camber originally placed in the rail. The contraction is resisted by the rigidity of the head and so exerts a tensile stress on the head, at least in its top surface, causing a resultant tensile stress to be set up in the base. However, because of the low strength and plastic nature of steel at temperatures above the $A_{3}$ transformation the stresses developed are probably very small.

According to Figure 30 the base enters the transformation range before the head and, according to Figure 38, expansion then occurs. This acts to relieve any tension that may be present in the base and, consequently, in the head. The transformation on the surface of the head occurs somewhat later than on the edge of the base (fig. 30). It, therefore, appears probable that the base being of lighter section passes completely through the transformation range before marked expansion will occur in the head. If this be true, the base will be contracting while the head is expanding incident to its cooling through the transformation range. The expansion of the head will tend to compensate for the contraction of the base, and so tend to prevent the developing of internal stresses. However, as soon as the head emerges from the transformation range, cooling of the head is relatively rapid, the head in fact, as is evident in Figures 30 and 31, cooling more rapidly than the base. The more rapid rate of cooling is accompanied by a more rapid contraction. This relatively high rate of contraction is resisted by the slower contracting web and base, which must simultaneously develop a tensile stress in the head and also acts to decrease the convexity of the rail. The base must then be placed in tension also, and consequently the web must be placed in compression. The locations of the neutral axes are indeterminate. They will depend, among other factors, upon the design of section and the relative rates of cooling of the head, web, and base. The head and base of the rail are, therefore, left in a state of internal tension and some central portion (the web) in compression. The exact distribution is not determinable.

In the preceding discussion no mention has been made of the temperature gradient that exists between the outside surface and central portion of the head. During cooling the center is always at a higher temperature, the difference depending upon the rate of cooling.

For convenience, consider the head separately from the web and base. As cooling occurs, the outside surface cools more rapidly than the inside. A temperature gradient is established. The outside will contract thereby tending to induce a compressive stress on the central portion of the head and resultant tensile stress on the outer fibers. Due to the low strength and high plasticity of steel at tem$98046^{\circ}-30-9$ 
peratures above the transformation range, these stresses will be very small. When the outside layers reach the $A_{3}$ transformation, expansion occurs which will relieve any tensile stress that may be present. The compression in the underlying metal is therefore relieved and possibly the metal placed into tension. As the underlying portions reach the transformation temperature, they, in turn, expand relieving any stress on them and in turn tend to pass a tensile stress on to the more central layers.

Just as the center emerges from the transformation range, it is evident from Figure 30 that its rate of cooling is faster than the rate of cooling of the surface. The rate of contraction of a central portion of the head is then greater than in the surface layers. A tensile stress is therefore developed in the central portion of the head which will be additive to any other tensile stresses that may be present at the same time. These stresses would be additive to those developed by the more rapid cooling of the base relative to the head.

It is evident from the above discussion that the stress distribution in a rail varies during the cooling in a very complex and indeterminate manner. The magnitude of the stresses can not be estimated without data regarding the temperature distribution during cooling, the modulus of elasticity of the steel during cooling, its yield point, and other properties. Transverse stresses would also be present, as suggested previously by Howard.

Certain facts appear significant, however. It has been shown that, as the head of a rail cools through the temperature range just below the $A r$ transformation, tensile stresses are developed in the head and base, due to differential cooling. From Figure 30 this range begins when the center is at about $670^{\circ} \mathrm{C}$. A maximum temperature difference between edge of base and center of rail was noted for air cooling when the center was at approximately $660^{\circ} \mathrm{C}$. and the edge of base at $470^{\circ} \mathrm{C}$. The base, at the latter temperature and below, will have a relatively high resistance to plastic deformation, especially as cooling enters the blue-heat range. On the other hand, the center of the head, at $660^{\circ} \mathrm{C}$., is entering the secondary brittle range. The tensile strength, elongation, and reduction of area of some rail steels are relatively low in this temperature range. (Fig. 3.) The steel is therefore in an unfavorable condition to resist the tensile stresses imposed, and rupture may conceivably occur. The somewhat higher temperature of the center and, consequently, somewhat lower strength and ductility during the period that stress is developed may account for failure occurring internally, giving rise to transverse shatter cracks. The added internal tensile stress incident to the temperature gradient between center and surface of the head is also probably a contributing cause for internal failure.

The marked difference in the degree of secondary brittleness in different heats, its marked intensity in heat No. 3 (fig. 3) from which a new rail was found to contain shatter cracks, ${ }^{26}$ its equally marked intensity in rail from heat PO (fig. 11) which failed by transverse fissure and showed shatter cracks, and its presence in rail from heat CN1 (fig. 12), which also showed shatter cracks, all tend to confirm the hypothesis that shatter cracks may be associated with secondary brittleness. 
That a rail may have a secondary brittleness and not show shatter cracks is indicated by rail from heat CN2. (Fig. 12.) This rail was subjected to identical service with rail from heat $\mathrm{CN} 1$, which failed by transverse fissure and contained shatter cracks. The two rails were from different heats and so were rolled and cooled at different times. Both steels had secondary brittleness and, therefore, probably had a propensity to form shatter cracks. The degree of secondary brittleness, however, was not very pronounced. The assumption seems reasonable that the rail (heat CN1) that developed shatter cracks may have been cooled at a relatively faster rate than the one from heat $\mathrm{CN} 2$ with the development of sufficiently higher stresses to cause rupture in the secondary brittle range. This indicates a rather critical "border-line" condition in cooling rates when secondary brittleness is prosent. Obviously, if all rails were cooled sufficiently slowly to prevent the formation of internal stresses, shatter cracks, which admittedly constitute the relief of internal strains, would not be formed. It also seems evident that a rail steel having very slight secondary brittleness, such as heat No. 21 (fig. 7), should be less liable to failure under conditions of relatively rapid cooling than a steel having marked secondary brittleness. The tensile strength of this steel (heat No. 21) is approximately the same in its secondary brittle range as some of the other rail steels, but due to its greater ductility in this temperature range considerable flow could occur and so relieve without rupturing any stress that might develop during cooling.

It is fully appreciated that the tensile properties of the steel of a rail at a given temperature during cooling after rolling may be different from the properties of the same material if allowed to cool and then reheated to the given temperature and tested. For temperatures above the transformation range the refinement of grain and other changes incident to the $A c$ transformations would very probably give different properties. That such a difference may exist at lower temperatures is indicated by the tests of specimens from heats No. 3 and $\mathrm{PO}$ which were annealed at a temperature $\left(700^{\circ} \mathrm{C}\right.$.) just below the $A c_{1}$ transformation; this annealing caused a marked decrease in the degree of secondary brittleness. This fact indicates that secondary brittleness may be more pronounced during cooling than after cooling and reheating to the secondary brittle range.

In the tests of specimens from medium manganese rails (fig. 15) it is evident that this steel shows marked secondary brittleness. It is known that steel of this type ${ }^{27}$ has given excellent service in track and is reported to be very free from shatter cracks and failures of the transverse-fissure type. This appears to contradict somewhat the hypothesis that shatter cracks (nuclei for transverse fissures) may be associated with secondary brittleness, especially as rails from these heats have been reported ${ }^{28}$ as giving good service in track. However, shatter cracks have been reported in intermediate manganese rail as the cause of horizontal crushed-head failures, and transverse fissures have also been reported in rail steel of this type ${ }^{29}$ It would be of interest to determine if such rail showed secondary brittleness. It is also true that shatter cracks are not necessarily

27 Report of Rail Committee, Bull. Am. Rwy. Eng. Assoc.; March, 1929.

28 Private communication from G. J. Ray; September, 1929.

29 See footnote 27. See data furnished by New York Central R, R. 
present in a rail showing secondary brittleness; their formation depends upon rate of cooling of the rail.

The fact that secondary brittleness is found in other steels than rail steels suggests that other types of unexplained failures, such as internal cracks in ingots or forgings, may be due to secondary brittleness. Hultgren ${ }^{30}$ has shown for a ball and roller bearing steel $(0.70 \mathrm{C}, 1.0 \mathrm{Cr}, 1.0 \mathrm{Mn})$ that internal cracks when present invariably formed during cooling after the final hot-working operation and that their formation is prevented by retarded cooling. He suggested that shatter zones in rails were of similar origin. It is suggested that the cause of the development of internal cracks in the steel studied by Hultgren might have been due to secondary brittleness in the steel.

The data indicate that equalization of temperature and slow cooling of rails or other steel objects through the secondary brittle range are desirable procedures to prevent formation of internal failure.

\section{PRELIMINARY STUDIES OF CAUSE OF SECONDARY BRITTLENESS}

It has been shown that secondary brittleness may be present in a relatively pure iron, rail steels, high-carbon steels, and alloy steels. The low ductility has been shown to be related apparently to a grain boundary condition of the steel as was indicated by the marked intercrystalline shattering found in specimens tested in the secondary brittle range. The cause then must be related to one or more of the following: Some structural condition, an element, or nonmetallic inclusions which may be present in steels of widely varying composition. The influence of free cementite has been suggested. The fact that annealing below the $A c_{1}$ transformation affects the degree of secondary brittleness indicates that it may be related to a constituent capable of solid solubility or precipitation at temperatures below the $A_{1}$ transformation.

Nitrogen analyses ${ }^{31}$ have shown no relation of nitrogen content to degree of secondary brittleness. A few residue analyses ${ }^{31}$ have been made and show no relation of degree of secondary brittleness to total $\mathrm{Al}_{2} \mathrm{O}_{3}$ or $\mathrm{SiO}_{2}$ content. Secondary brittleness has been found in rail steels killed with aluminum and also in rail steels in which aluminum was not used. Due to difficulties in analytical methods for the determination of $\mathrm{MnO}$ or manganous silicate inclusions an indicated relation to $\mathrm{MnO}$ content can not yet be considered significant. Space is not available to report in detail on this phase of the problem at the present time. It may be stated that detailed studies along the lines suggested are being carried out.

\section{SUMMARY AND CONCLUSIONS}

A study has been made of the tensile properties of rail steels and some other steels at elevated temperatures. Special study was made of the tensile properties in the approximate temperature range of $400^{\circ}$ to $700^{\circ} \mathrm{C}$. It has been found that the ductility of all rail steels,

${ }^{80}$ Hultgren. Arel. Flakes or Hair Cracks in Chromium Steels with a Discussion of Shattered Zones and Transverse Fissures in Rails, J. Iron and Steel Inst., 111, No. 1, p. 113, 1925.

si By J. S. Acken, Bureau of Standards. 
as measured by percentage elongation and reduction of area, decreases with increase in temperature over a portion of this temperature range. In some instances the decrease in ductility is very marked, the elongation and reduction of area values becoming less between approximately $500^{\circ}$ to $650^{\circ} \mathrm{C}$. than at normal atmospheric temperatures. The same phenomena have been found to exist in Armco iron, a 1 per cent carbon steel, and some alloy steels. This range of temperature in which low ductility becomes manifest has been termed the "secondary brittle" range to distinguish it from the wellknown "blue brittle" range occurring at $200^{\circ}$ to $300^{\circ} \mathrm{C}$. and the "hot-short" range which is usually associated with the $A_{3}$ transformation and high sulphur content. The phenomenon has been termed "secondary brittleness."

The degree of secondary brittleness varies markedly between individual heats and the temperature of the maximum effect also varies.

The data indicate that the secondary brittleness is not confined to individual rails or ingots, but is apparently a property of the heat as a whole. It has been shown that the degree of secondary brittleness is decreased by annealing at a temperature $\left(700^{\circ} \mathrm{C}\right.$.) slightly below the $A c_{1}$ transformation as well as at the usual annealing temperatures above the $A c_{3}$ transformation.

The cause of secondary brittleness has not been determined. Residue analyses and analyses for nitrogen content indicate that the phenomenon is not related to silica, alumina, or nitrogen contents. Some evidence has been obtained which indicates it may be related to the $\mathrm{MnO}$ content or free carbides.

Data are also given in the report on the rate of cooling and temperature distribution in a 130-pound $\mathrm{P}$. S. section rail during cooling in still air, moving air, and during quenching in cold and hot water.

An hypothesis as to the formation of shatter cracks in rails, based on the phenomenon of secondary brittleness and temperature distribution during cooling, is presented. Rapid cooling through the secondary brittle range is believed to cause the formation of shatter cracks in rails showing secondary brittleness. Shatter cracks are believed by many to be the nucleus of transverse fissures. It is therefore suggested that the origin of transverse fissures is related to secondary brittleness and rate of cooling of the rail.

The fact that secondary brittleness is found in other steels than rail steels suggests that other types of unexplained failures, such as internal cracks in ingots or forgings, may be due to secondary brittleness.

The data indicate that equalization of temperature and slow cooling of rails and other steel shapes through their secondary brittle range are desirable procedures to follow in order to prevent formation of shatter cracks.

Washington, October 19, 1929. 